\title{
Shear Stability of a Vegetable Oil-Based Lubricant with Ethyl Cellulose Viscosity Index Improver
}

by

\author{
Niall McCallum \\ A thesis submitted to \\ the Faculty of Graduate and Postdoctoral Affairs \\ in partial fulfilment of \\ the requirements for the degree of \\ Master of Applied Science \\ in \\ Mechanical Engineering
}

Ottawa-Carleton Institute for Mechanical and Aerospace Engineering

Department of Mechanical and Aerospace Engineering

Carleton University

Ottawa, Ontario, Canada

April 12, 2018

Copyright (c)

2018 - Niall McCallum 


\section{Abstract}

A diesel fuel injector apparatus conforming to ASTM 6278 was used to examine the effects of mechanical shear on two vegetable oil-base lubricants, each blended with a different molecular weight ethyl cellulose polymer to improve its viscosity-temperature behaviour. Kinematic viscosity measurements conforming to ASTM D445 were used to determine the magnitude of viscosity loss after mechanical shearing. The lower molecular weight E45 sample was determined to have a permanent viscosity loss of $0.73 \%$ and a shear stability index of 1.56 , whereas the higher molecular weight EC100 sample was determined to have a permanent viscosity loss of $3.57 \%$ and a shear stability index of 6.51. Results were comparable to traditional PMMA polymer additives of similar molecular weight which are commonly used in petroleum-based lubricants, indicating that the ethyl cellulose polymers may function well as VIIs even after mechanical shear degradation. Kinematic viscosity measurements of increasingly dilute solutions of prepared lubricants were used to determine the changes in the intrinsic viscosity of solution as an indicator of the decrease in polymer molecular weight after shearing. 


\section{Table of Contents}

Abstract $\quad$ ii

Table of Contents iii

List of Tables $\quad$ V

List of Figures viii

List of Symbols $\quad x$

1 Introduction and Problem Statement 1

2 Literature Review 3

2.1 Environmental Impacts of Lubricating Oils . . . . . . . . . . . . . . 3

2.2 Biodegradable Lubricants . . . . . . . . . . . . . . . 6

2.2.1 Base Oil Properties . . . . . . . . . . . . . . . 6

2.2.2 Vegetable Oils for Lubricant Use . . . . . . . . . . . . . . . 7

2.3 Physical Properties of Lubricating Oils . . . . . . . . . . . . . . . 14

2.3.1 Lubricant Viscosity . . . . . . . . . . . . . . . . . . . . . . 14

2.3.2 Viscosity Additives . . . . . . . . . . . . . . . . . . . . . . 19

2.3.3 Solution Properties ................. 23

2.3.4 Permanent Viscosity Loss due to Mechanical Shear . . . . . . 24

2.4 Experimental Methods in Mechanical Degradation of Polymers . . . . 26 
3 Experimental Setup $\quad 29$

3.1 Materials . . . . . . . . . . . . . . . . 29

3.2 Preparation of Lubricant Blends . . . . . . . . . . . . . . . . . . . 30

3.3 Shearing Apparatus . . . . . . . . . . . . . . . . . . 32

3.4 Viscosity Measurements . . . . . . . . . . . . . . . 37

3.4.1 Determination of Kinematic Viscosity . . . . . . . . . . . . . 38

3.4.2 Determination of Intrinsic Viscosity . . . . . . . . . . . . . . 40

3.5 Determination of Solution Density . . . . . . . . . . . . . . 41

4 Experimental Results $\quad 44$

4.1 Calibration with RL233 Reference Oil . . . . . . . . . . . . . . . . 44

4.2 Shear Stability of Blended Lubricants . . . . . . . . . . . . . . 45

5 Conclusions and Recommendations for Future Work 55

5.1 Summary of Experimental Results . . . . . . . . . . . . . 55

5.2 Recommendations for Future Work . . . . . . . . . . . . . 56

$\begin{array}{ll}\text { References } & 58\end{array}$

Appendix A Dilution Plots for Determination of Intrinsic Viscosity 63

$\begin{array}{lll}\text { Appendix B MATLAB Curve Fit } & 68\end{array}$ 


\section{List of Tables}

2.1 Primary and ready biodegradability of lubricant base fluids [1] . . . .

2.2 Comparison of selected parameters for environmentally acceptable lubricating oils $[2] \ldots \ldots \ldots \ldots \ldots \ldots$

2.3 The kinematic viscosity and viscosity index of a blended base oil may be varied by changing the ratio of high-oleic sunflower oil (HOSO) and castor oil (CO). Blends were also thickened with a 1 wt.\% viscosity index additive $[3] \ldots \ldots \ldots \ldots$. . . . . . . . . . . . . . . . . 14

2.4 The addition of an ethyl cellulose polymer increased the viscosity and viscosity index compared to the base oil [3] . . . . . . . . . . 23

2.5 Viscometric terminology . . . . . . . . . . . . . . 23

3.1 Fatty acid composition (as provided by manufacturer data sheets) of the vegetable oils used in this research. . . . . . . . . . . . 30

3.2 Physical properties of polymer additives . . . . . . . . . . . . 30

3.3 Properties of base oils and formulated lubricants with $1 \%$ wt. ethyl cellulose polymer additives. EC45 denotes a lubricant blend with ethyl cellulose $(45 \mathrm{cP})$ and EC100 denotes a lubricant blend with ethyl cellulose $(100 \mathrm{cP}) \ldots \ldots \ldots \ldots$ 
4.1 Summary of calibration tests with reference oil RL233 after 30 shear cycles (30 SC) through the injector apparatus. A nozzle pressure of 2200 psi produced a sufficient amount of degradation in the reference oil to proceed with shear stability testing of blended lubricants. . . .

4.2 Summary of kinematic viscosity changes for blended lubricants after 30 shear cycles (30 SC). Comparison results are also shown for a study which investigated the shear degradation of four separate molecular weights of PMMA (identified as MA1 to MD1 in the study) additive

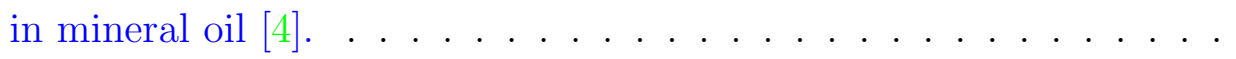

4.3 Kinematic and dynamic viscosities of diluted lubricant samples with ethyl cellulose (EC) viscosity index additives before and after 30 cycles (30 SC) of mechanical degradation . . . . . . . . . . . . .

4.4 Relative and specific viscosities of diluted lubricant samples with ethyl cellulose (EC) viscosity index additives before and after 30 cycles (30 SC) of mechanical degradation . . . . . . . . . . . . . . .

4.5 Intrinsic viscosity as determined by a linear regression of reduced viscosity measurements on diluted lubricant samples with ethyl cellulose (EC) viscosity index additives before and after 30 cycles (30 SC) of mechanical degradation . . . . . . . . . . . . . . . .

4.6 Intrinsic viscosity as determined by a linear regression of inherent viscosity measurements on diluted lubricant samples with ethyl cellulose (EC) viscosity index additives before and after 30 cycles (30 SC) of mechanical degradation . . . . . . . . . . . . . . . . 
4.7 Huggins coefficients and intrinsic viscosity of diluted lubricant samples with ethyl cellulose (EC) viscosity index additives before and after 30 cycles (30 SC) of mechanical degradation. Coefficients were estimated from a second order polynomial curve fit of the specific viscosity based

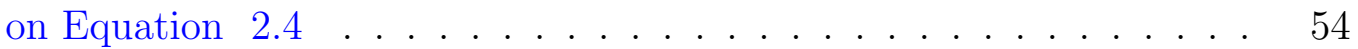

4.8 Kraemer coefficients and intrinsic viscosity of diluted lubricant samples with ethyl cellulose (EC) viscosity index additives before and after 30 cycles (30 SC) of mechanical degradation. Coefficients were estimated from a second order polynomial curve fit of the relative viscosity based on the Equation $2.5 \ldots \ldots \ldots \ldots \ldots$

4.9 Percent decrease of the intrinsic viscosity of blended lubricants after shearing as determined by a second order polynomial curve fit of the Huggins and Kraemer equations, and a linear approximation (ASTM

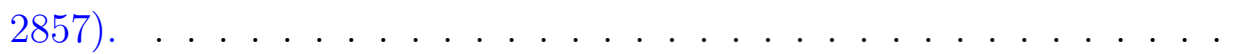




\section{List of Figures}

2.1 Representation of a triglyceride molecule [5] . . . . . . . . . . . . 8

2.2 Chemical structure of a saturated fatty acid $[5] \ldots \ldots$. . . . . . 9

2.3 Chemical structure of a monounsaturated fatty acid [5] . . . . . . . 10

2.4 Chemical structure of a polyunsaturated fatty acid [5] . . . . . . 10

2.5 Chemical structure of a hydroxy fatty acid [5] . . . . . . . . . . 11

2.6 Chemical structure of oleic acid $[6] \ldots \ldots$. . . . . . . . . . . 12

2.7 Chemical structure of ricinoleic acid [6] . . . . . . . . . . . . 13

2.8 Schematic of two parallel plates in relative motion separated by a thin fluid film $[5] \ldots \ldots \ldots$. . . . . . . . . . . . . . . . 15

2.9 Viscosity - temperature relationship for several SAE grade oils [7] . . 18

2.10 Viscosity-temperature relationship of different VI grade lubricants . . 19

2.11 The addition of high molecular weight polymers can improve the viscosity-temperature performance of blended lubricants . . . . . . . 20

2.12 The polymer coil dimensions may increase or decrease with variations in temperature, as measured by changes in the reduced viscosity of a polymer solution containing (a) polyalkylmethacrylate, (b) ethylenepropylene copolymer, and (c) styrene-butadiene copolymer [7]. . . . .

2.13 The addition of an ethyl cellulose polymer increased the film thickness compared to the base oil. Measured at (a) $40^{\circ} \mathrm{C}$ and (b) $100^{\circ} \mathrm{C}$ [3]. . 
2.14 Schematic representation of the permanent degradation of a polymer

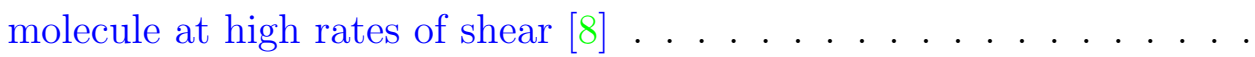

2.15 Changes in the kinematic viscosity measured at $100^{\circ} \mathrm{C}$ for lubricant blends sheared with the diesel injector test (top row) and the sonic shear stability test (bottom row) [9] . . . . . . . . . . . . . . 28

3.1 Details of the injector pump body [10]. . . . . . . . . . . . .

3.2 Schematic of the modified injector assembly. White arrows indicate the pneumatic subassembly and solid arrows indicate the flow of sample oil through the apparatus . . . . . . . . . . . . . . 35

3.3 Photo of the modified injector assembly with key components labelled. 36

3.4 Schematic of the oil bath as specified by ASTMD445 [11] . . . . . . . 39

3.5 Example plot constructed to determine the intrinsic viscosity of polymer containing solutions [12] . . . . . . . . . . . . . . 41

3.6 Calibrated Gay-Lussac density bottles were used for determination of fluid density at $40^{\circ} \mathrm{C}$ and $100^{\circ} \mathrm{C}$. . . . . . . . . . . . . . 43

4.1 Relative viscosity of diluted sheared and unsheared samples at $40^{\circ} \mathrm{C} . \quad 50$

4.2 Relative viscosity of diluted sheared and unsheared samples at $100^{\circ} \mathrm{C} \quad 50$

A.1 EC100 Dilution at $40^{\circ} \mathrm{C} \ldots \ldots \ldots \ldots$

A.2 EC100 Dilution at $100^{\circ} \mathrm{C} \ldots \ldots \ldots$. . . . . . . . . 64

A.3 EC100 30SC Dilution at $40^{\circ} \mathrm{C} \ldots \ldots . \ldots . \ldots . \ldots 64$

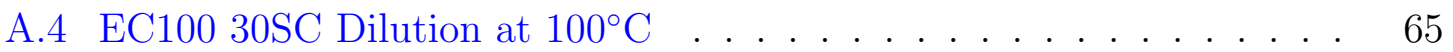

A.5 EC45 Dilution at $40^{\circ} \mathrm{C} \ldots \ldots \ldots \ldots$

A.6 EC45 Dilution at $100^{\circ} \mathrm{C} \ldots \ldots \ldots 6$

A.7 EC45 30SC Dilution at $40^{\circ} \mathrm{C} \ldots \ldots \ldots 6$

A.8 EC45 30SC Dilution at $100^{\circ} \mathrm{C} \ldots \ldots \ldots$. . . . . . . . 67 


\section{List of Symbols}

\begin{tabular}{ll}
\hline Nomenclature & Definition \\
\hline $\mathrm{c}$ & solution concentration (\% weight or $\left.\mathrm{g} / \mathrm{cm}^{3}\right)$ \\
$\mathrm{cSt}$ & Castor oil \\
$\mathrm{cP}$ & centistokes \\
EVA & centipoise \\
HOSO & Ethylene-vinyl acetate copolymer \\
PAG & High-oleic sunflower oil \\
PAO & Polyalkylene glycol \\
PMMA & Polyalphaolefins \\
psi $(\mathrm{g})$ & Poly(methyl methacrylate) \\
PVL & pounds per square inch (gauge) \\
SC & Permanent Viscosity Loss \\
SBS & Shear Cycles \\
SSI & poly(styrene-butadiene-styrene) copolymer \\
TSS & Shear Stability Index \\
VI & Total Sum of Squares \\
VII & Viscosity Index \\
$\eta$ & Viscosity Index Improver \\
$\eta_{0}$ & Dynamic (absolute) viscosity (cP) \\
$\nu$ & Dynamic viscosity of base oils \\
$\nu_{0}$ & Kinematic viscosity (cSt) \\
\hline &
\end{tabular}




\section{Chapter 1}

\section{Introduction and Problem Statement}

Lubricants form an essential component of machinery by reducing wear and energy loss associated with friction between sliding or rotating components. Lubricating oils also protect machinery and equipment by transporting heat and wear debris away from the contact site where it can be removed from the system [5]. While there are many different types of lubricants formulated to suit the specific needs of an application, most industrial lubricants contain a base oil which is derived from petroleum base stocks and blended with additives to improve its chemical and physical properties for a given set of operating conditions [13].

One of the most important physical properties of any lubricant is its viscosity at operating temperature. A lubricant must have sufficient viscosity to ensure there is an adequate film thickness separating surfaces in relative motion. Where the base oil viscosity is too low at a given operating temperature, or where the decrease in viscosity is too great across a range of temperatures, viscosity index additives are blended in solution with the base oil to improve its viscosity-temperature characteristics. These viscosity additives are typically long-chain polymer molecules which may fragment when the lubricant is subjected to high rates of shear - effectively altering the physical properties of the blended lubricant [14]. 
Although petroleum-based lubricants are well understood by researchers and industry, serious consideration for biodegradable or environmentally friendly lubricants has only been given in response to an increasing awareness of our ability to cause long term or permanent environmental degradation from certain industrial practices. The scope of this experimental research was to promote the qualification of biodegradable lubricants for greater industrial use by examining the shear stability of non-toxic and biodegradable lubricants blended with polymer viscosity index additives. 


\section{Chapter 2}

\section{Literature Review}

\subsection{Environmental Impacts of Lubricating Oils}

Lubricating oils derived from plants or animal fats were used throughout human history. Since these early lubricants were derived from renewable and biodegradable sources, their impact on the environment in the event of a spill was minimal. With the discovery of petroleum in the 19th century, naturally derived lubricants were replaced in most applications with blends derived from mineral oil. [15]

The increased availability and improved performance of petroleum based or synthetic lubricants limited the use of bio-lubricants to a few specialized industries where environmental concerns take precedent over performance or cost. These may be total loss applications where the lubricant is completely discharged into the environment during use, or where applications involve activity in environmentally sensitive areas that would be harmed by lubricant leaks or spills. Some typical uses for biodegradable lubricants include : [16]

1. Outboard marine two-stroke engine oils 
2. Chainsaw and saw frame oils

3. Hydraulic oils for construction machinery and forestry equipment

4. Lubricants for food machinery

5. Lubricants for metalworking and machining

Although a detailed lifecycle analysis of the environmental impact of lubricants is beyond the scope of this work, it is possible to generalize on the differences in environmental impact between mineral oil-based and biodegradable lubricants during production and end of life. During production, the typical energy consumption for a mineral oil-based lubricant has been estimated at $9 \mathrm{MJ}$ per $\mathrm{kg}$ of lubricant [17] whereas a biodegradable vegetable oil-based lubricant may require as little as $3 \mathrm{MJ}$ per $\mathrm{kg}$ of lubricant produced [18]. Manufacturing a lubricant from naturally derived carbon also helps to reduce the amount of new carbon released into the atmosphere.

Perhaps the greatest environmental impact occurs during use or at the end of life where lubricants may spill or not be properly disposed of. It is estimated that approximately 1.3 million tonnes of petroleum pollutants may be discharged into the world's oceans annually [19]. If used petroleum-based lubricants are released into environmentally sensitive marine habitats, plant life may experience decreased photosynthesis and nutrient availability, and the used lubricating oil could destroy root tissue [20]. As much as 50\% of all lubricants end up in the environment through loss in application, spills, or illegal dumping [21]. In applications with biodegradable lubricants, approximately 12 million tonnes annually are released into the environment [16] 
The degree of biodegradability is largely dependent on the type of base oil, although the type and formulation of performance additives can also affect the toxicity and biodegradability of formulated lubricants [22]. The degree of biodegradability is normally assessed using two different types of tests to determine the primary biodegradability and the ultimate biodegradability [1]. The primary biodegradability describes how quickly oils are transformed into carboxylated or hydroxylated intermediates which have a greater water solubility, and is determined by the CECL-33-A-93 test [23]. Ultimate biodegradability is a measure of how long a lubricant may persist in the environment before it is completely degraded. The OECD 301 test has been developed to measure the ultimate biodegradability by monitoring the production of carbon dioxide from a flask containing a solution of the test substance in a mineral medium [24]. Typical values for these tests are listed in Table 2.1 below [1].

Table 2.1: Primary and ready biodegradability of lubricant base fluids [1]

\begin{tabular}{rcc}
\multicolumn{1}{c}{$\begin{array}{c}\text { Lubricant } \\
\text { Type }\end{array}$} & $\begin{array}{c}\text { Primary Biodegradability } \\
\text { (CEC-L-33-A-94) 21 days }\end{array}$ & $\begin{array}{c}\text { Ready Biodegradability (OECD } \\
\text { 301B) 28 days }\end{array}$ \\
\hline Mineral Oil & $10-45$ & $10-40$ \\
PAO & $20-80$ & $5-60$ \\
Alkylbenzene & $5-20$ & $0-20$ \\
Diester (aliphatic) & $75-100$ & $50-95$ \\
Polyol Ester & $0-100$ & $0-85$ \\
Vegetable Oil & $90-100$ & $75-95$
\end{tabular}

The use and disposal of lubricating oils has the potential for significant environmental impact. With advances in production technology and increased demand for environmentally sustainable industrial practices, the study of biodegradable lubricants addresses an important need to evaluate the lifecycle of a product or process and reduce the impact of human activity. [25] 


\section{$2.2 \quad$ Biodegradable Lubricants}

The definition of a bio-lubricant may vary depending on the industry or intended use. The terms green lubricants, environmentally acceptable lubricant, or environmentally friendly lubricant are generally used interchangeably by manufacturers, and care must be taken to adequately define the desired properties before selecting a lubricating oil for environmentally sensitive applications. While certain lubricant blends may be non-toxic or environmentally benign, the requirements for a completely biodegradable and renewable lubricant presents interesting opportunities for research as they are expected to become more prevalent with industrial agriculture, forestry, and water treatment. [5] [26]

Biodegradable lubricants derive many of their properties from the inherent biodegradability of their base oils [27]. Even where petroleum based lubricants are free from zinc and calcium performance additives (which are generally considered to be toxic to human health and to the environment [28]), any petroleum-based lubricant is unable to be readily degraded and will persist in the environment for months or years. [2]

\subsubsection{Base Oil Properties}

The properties of a biodegradable lubricant are determined primarily from the structure and composition of its base oils. In most applications requiring the use of biodegradable or non-toxic lubricants, base oils may be derived from one of four sources [29].

1. Triglycerides

2. Polyglycols

3. Synthetic esters 


\section{Polyalphaolefins}

Where formulating a bio-lubricant, the specific requirements of the application must be considered. Biodegradable lubricants require careful selection in order to maximize their useful life in application. Athough a detailed overview of lubricant base oil properties and formulation is beyond the scope of this work, the general properties of environmentally friendly base oils are described in Table 2.2 [2].

Table 2.2: Comparison of selected parameters for environmentally acceptable lubricating oils [2]

\begin{tabular}{lllll} 
Parameter & Triglyceride & Synthetic Ester & PAG & PAO \\
\hline Renewability content & High & Variable & None & None \\
Biodegradability & Good & Good & Moderate & Moderate \\
Water solubility & Low & Low & Compatible & Low \\
Low-temperature performance & Weak & Good & Good & Good \\
Oxidation Stability & Weak & Good & Good & Good \\
Hydrolytic Stability & Low & Medium & Good & Good \\
Seal material compatibility & Good & Limited & Limited & Good \\
Paint and varnish compatibility & Good & Good & Limited & Good \\
Additive solubility & Good & Good & Moderate & Limited \\
Lubricity of base oil & Good & Good & Good & Limited \\
Corrosion Resistance & Poor & Limited & Limited & Good
\end{tabular}

\subsubsection{Vegetable Oils for Lubricant Use}

With a combination of biodegradability, renewability, and decreased wear under certain conditions, vegetable oil-based lubricants have seen increased attention from researchers as an environmentally friendly alternative to petroleum or synthetic lubricants. [3] [5]. Bio-lubricants sourced from renewable vegetable oils contain naturally occuring triglycerides which are inherently biodegradable and non-toxic [30]. 
Triglycerides are composed of a glycerin molecule linked to three fatty acid branches - a schematic of which is shown in Figure 2.1. The properties of a vegetable oil-based lubricant are primarily determined by the structure of its component triglyceride, which account for approximately $98 \%$ by weight of the molecular composition of vegetable oils and can vary significantly depending on the type of plant and region of origin [31] [2].

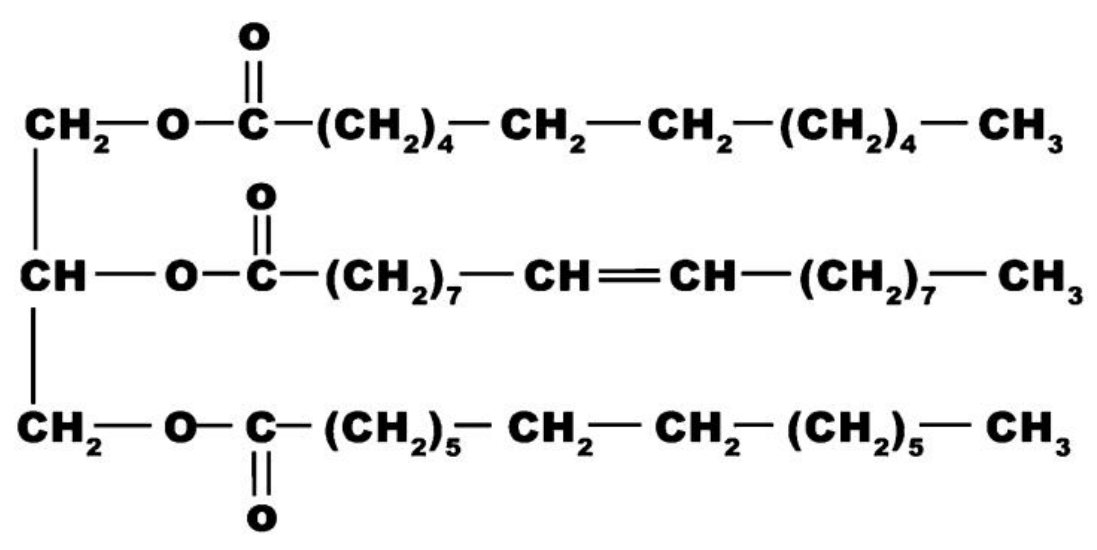

Figure 2.1: Representation of a triglyceride molecule [5]

Triglycerides may be characterized based on the degree of saturation. [5]

1. Saturated fatty acids (Figure 2.2) : Have no carbon-carbon double bonds in their structure. Examples include lauric acid, palmitic acid, and stearic acid.

2. Mono-unsaturated fatty acids (Figure 2.3) : Have one carbon-carbon double bond in their structure Examples include oleic acid and palmitoleic acid 
3. Poly-unsaturated fatty acids (Figure 2.4) : Have multiple carbon-carbon double bonds in their structure. Examples include linoleic acid and linolenic acid

4. Hydroxy fatty acids (Figure 2.5) : Have a hydroxyl group (-OH) in their structure

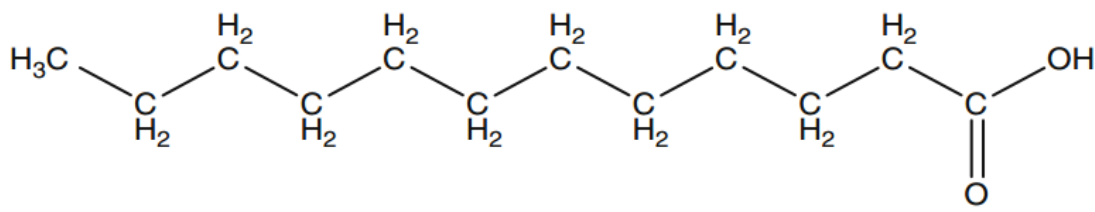

Figure 2.2: Chemical structure of a saturated fatty acid [5] 


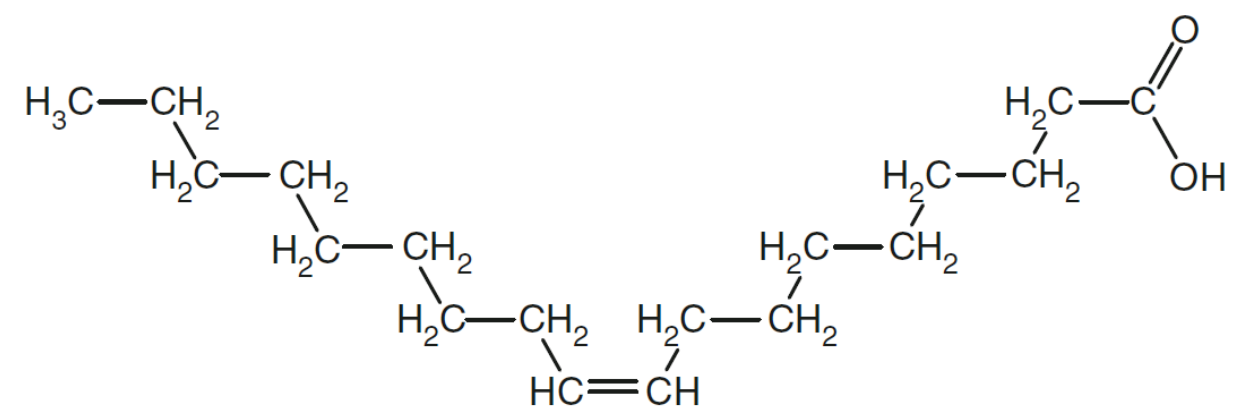

Figure 2.3: Chemical structure of a monounsaturated fatty acid [5]

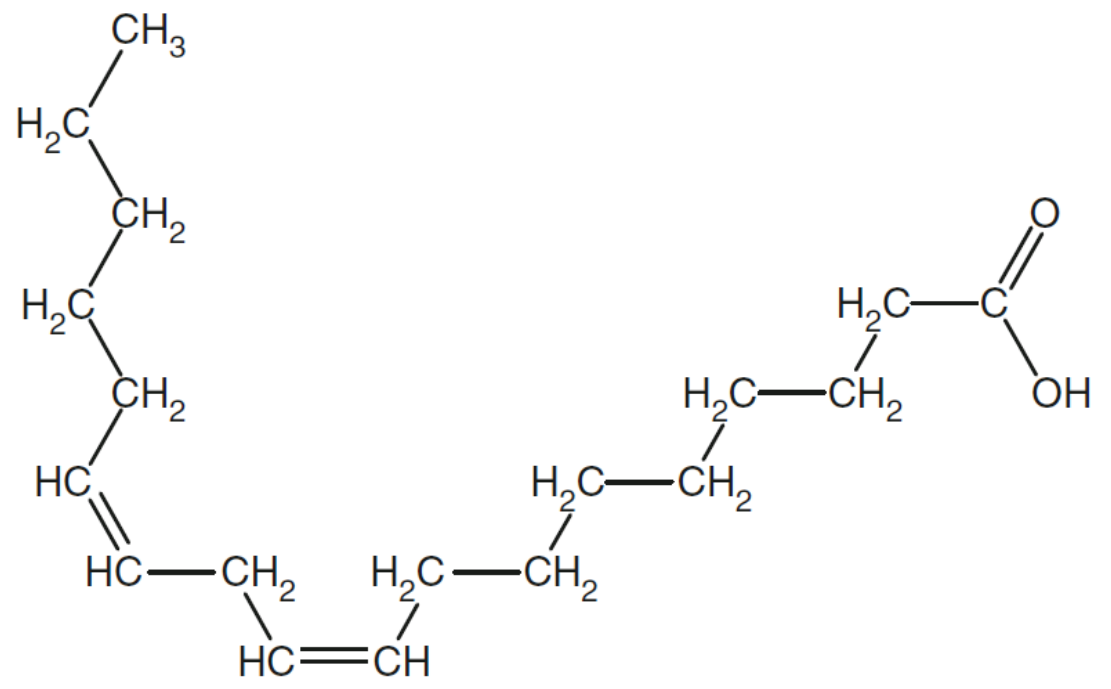

Figure 2.4: Chemical structure of a polyunsaturated fatty acid [5] 


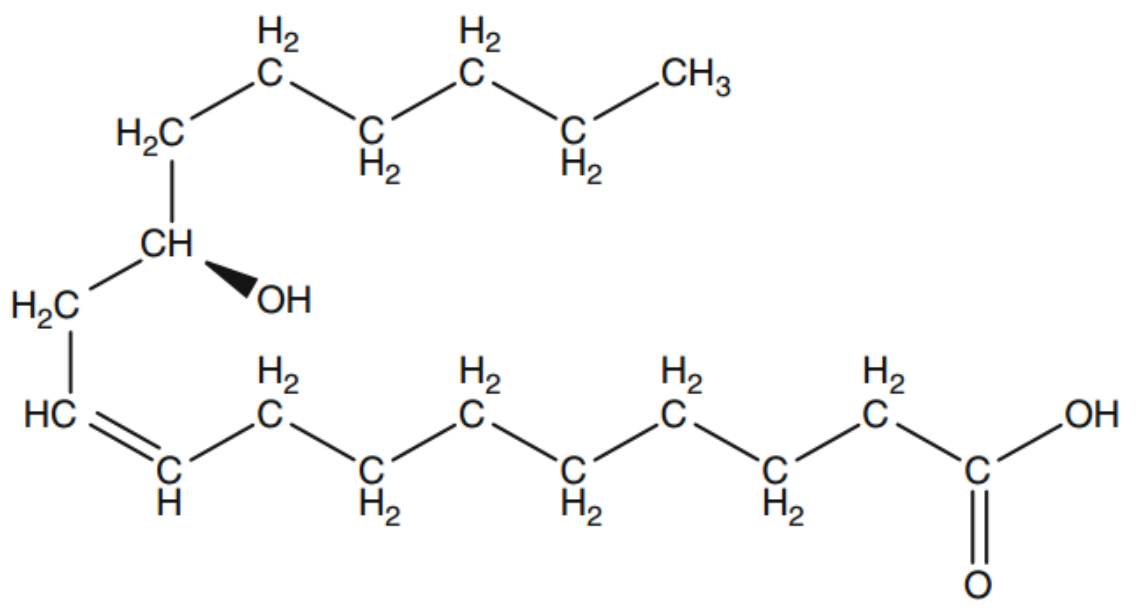

Figure 2.5: Chemical structure of a hydroxy fatty acid [5]

Variations in the performance properties of a vegetable oil-based lubricant are largely determined by the degree of unsaturation in the constituent triglycerides [2]. The presence of a double bond in mono- or poly-unsaturated fatty acids lowers the pour point and improves cold temperature performance [32]. However, the presence of carbon-carbon double bonds also introduces a potential reaction site and effectively lowers the oxidative stability of the lubricant at higher temperatures above $120^{\circ}$ C [33]. The most commonly used vegetable oils are high in monounsaturated or hydroxy fatty acids, as these strike a balance between cold temperature performance, oxidative stability, and a high viscosity index [34]. Vegetable oils can also possess natural anti-wear properties due to polar interactions with lubricated metal surfaces [34] [35].

Two notable examples that appear in literature are castor oil and high-oleic sunflower oil. Triglycerides in castor oil are primarily composed of ricinoleic acid (shown in 
Figure 2.7) which contains a free hydroxyl group that increases molecular polarity and is able to interact with metal surfaces to improve lubricity, reduce friction, and increase the solubility of polymer additives [32] [36] [3]. High oleic sunflower oil is harvested from genetically modified plants which contain a high concentration of oleic acid. Oleic acid (shown in Figure 2.6) is a monounsaturated fatty acid with a very consistent molecular structure which improves the viscosity index and oxidative stability of formulated lubricants [37].

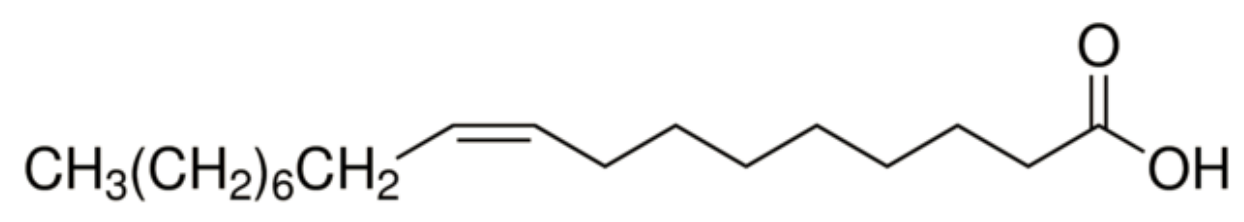

Figure 2.6: Chemical structure of oleic acid [6] 


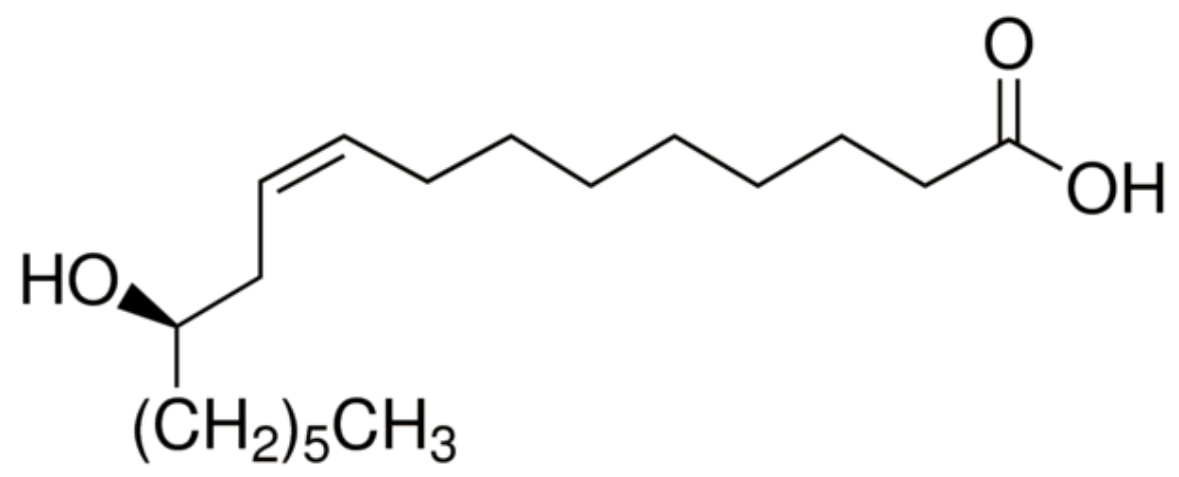

Figure 2.7: Chemical structure of ricinoleic acid [6]

It has been suggested in some studies that blends containing high-oleic sunflower oil and castor oil could combine the high viscosity index and oxidative stability of high-oleic sunflower oil with the superior lubricity of castor oil [3]. By varying the ratio of each base oil, it is possible to formulate a lubricant with a range of viscosities for use in different applications as shown in Table 2.3 [3]. 
Table 2.3: The kinematic viscosity and viscosity index of a blended base oil may be varied by changing the ratio of high-oleic sunflower oil (HOSO) and castor oil (CO). Blends were also thickened with a 1 wt.\% viscosity index additive [3]

\begin{tabular}{c|cccc} 
& \multicolumn{4}{|c}{ Kinematic Viscosity } \\
Blend & $40^{\circ} \mathrm{C}$ & $100^{\circ} \mathrm{C}$ & Viscosity Index & ISO Grade \\
\hline HOSO/CO (9:1) & 62 & 17 & 284 & ISO VG68 \\
HOSO/CO (7:3) & 95 & 22 & 264 & ISO VG100 \\
HOSO/CO (1:1) & 174 & 26 & 185 & ISO VG150 \\
HOSO/CO (3:7) & 293 & 29 & 134 & ISO VG320 \\
HOSO/CO (1:9) & 493 & 39 & 122 & ISO VG460
\end{tabular}

\subsection{Physical Properties of Lubricating Oils}

As previously discussed, the physical properties of lubricants are determined by the chemical composition of the base oil or additives. Although the chemical and oxidative stability of a lubricant is justifiably important, the scope of this research was to investigate physical changes in the properties of lubricating oils. The following section describes the physical and solution properties of formulated lubricating oils.

\subsubsection{Lubricant Viscosity}

Aside from the choice of base oil chemistry which may depend on the type of industry or application, lubricants are primarily selected based on the required viscosity at operating temperature. Viscosity is directly related to the ability for a lubricant to maintain adequate film thickness and separation between moving surfaces [5]. Generally speaking, if the sliding or rotational speeds are high or the machine operates in low temperatures, a low viscosity lubricant may be required. For low 
speed, high temperatures, or high load applications, a higher viscosity lubricant may be required to maintain adequate separation. [38]

Consider two parallel surfaces as shown in Figure 2.8 separated by a fluid film with a thickness $h$. A force $F$ is required to move the upper surface with velocity $u$. This force is proportional to the surface area of the upper surface $A$, and the sliding velocity $u$. Where the fluid is assumed to behave as an infinite number of thin sliding layers, a constant of proportionality is required to equate the applied force to the sliding velocity as shown in Equation 2.1 .

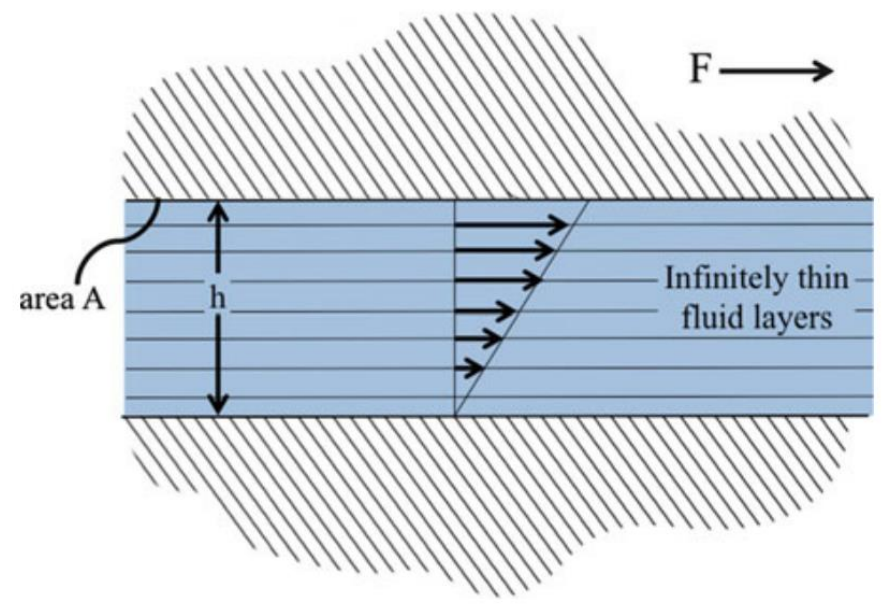

Figure 2.8: Schematic of two parallel plates in relative motion separated by a thin fluid film [5]

$$
F=\eta A \frac{u}{h}
$$

The dynamic (absolute) viscosity $\eta$ may be described as a resistance to flow - a 
proportionality constant that describes the relationship between shear stress and shear strain rate of a fluid element as described in Equation 2.2 [5]. The greater the dynamic viscosity, the greater the resistance to fluid motion. It may also be thought of as a macroscopic measurement which characterizes the microscopic interactions between fluid molecules moving relative to each other [8].

Rearranging Equation 2.1 to solve for fluid viscosity $\eta$

$$
\eta=\frac{(F / A)}{(u / h)}=\frac{\tau}{\dot{\gamma}}
$$

Where $\tau$ is the fluid shear stress $(\mathrm{Pa})$ and $\dot{\gamma}$ is the rate of shear $\left(\mathrm{s}^{-1}\right)$. The unit of dynamic viscosity is the pascal-second $(\mathrm{Pa} \cdot s)$, but is normally expressed in centipoise $(\mathrm{cP})$ for convenience where $1 c \mathrm{P}=10^{-3} \mathrm{~Pa} \cdot \mathrm{s}$

The kinematic viscosity is defined as the ratio of a fluid's dynamic viscosity to its density.

$$
\nu=\frac{\eta}{\rho}
$$

Where $\rho$ is the fluid density. The unit of kinematic viscosity is $m^{2} / s$, but is normally expressed in terms of centistokes (cSt) where $1 \mathrm{cSt}=10^{-6} \mathrm{~m}^{2} / \mathrm{s}$

Viscosity may vary with pressure or the rate of shear [39]. However, under normal operating conditions of industrial machinery, temperature has the greatest influence on lubricant viscosity. [5] The general trend is shown in Figure 2.9, where the viscosity is shown to decrease with an increase in temperature for several different 
SAE viscosity grade lubricants [7]. 


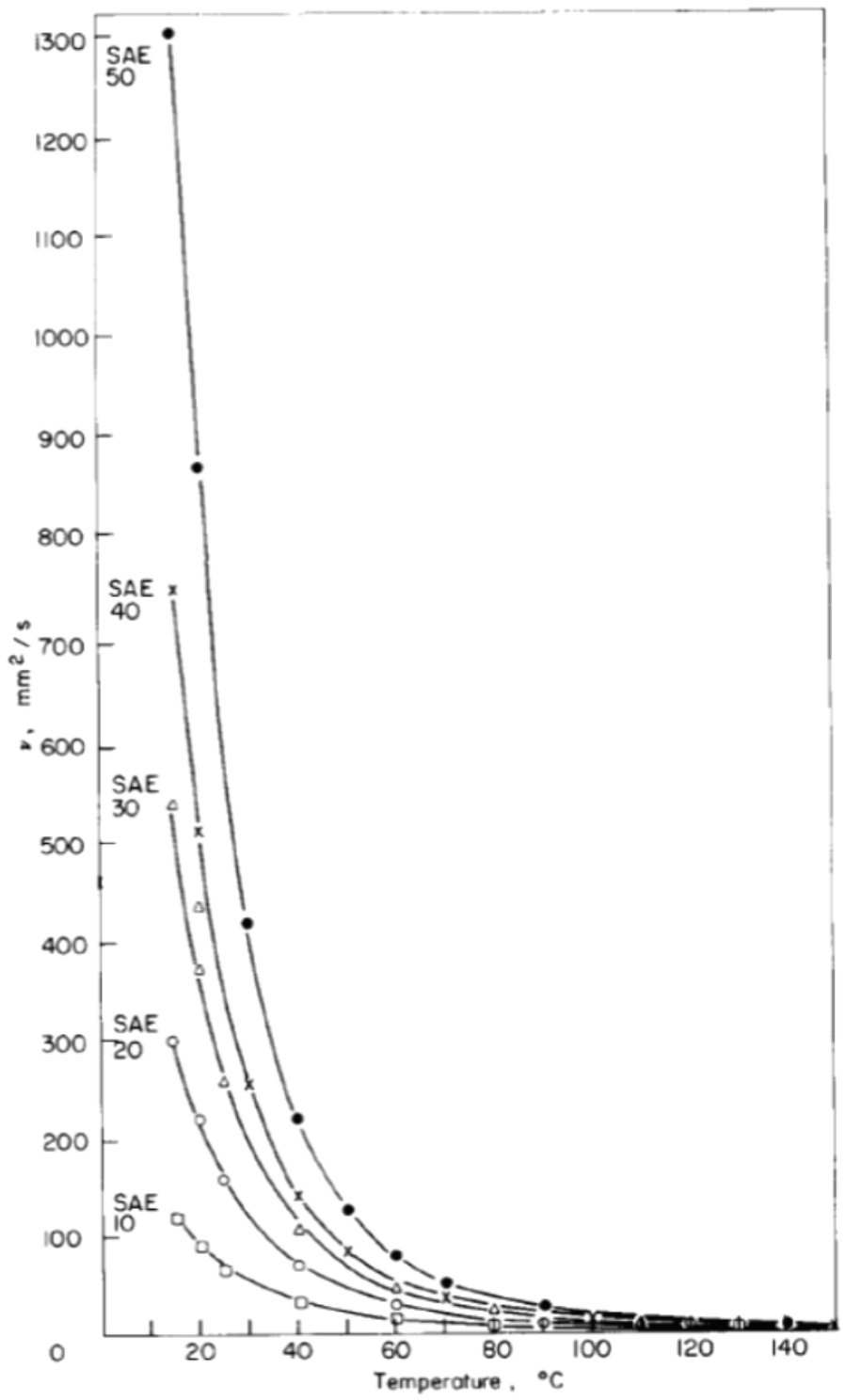

Figure 2.9: Viscosity - temperature relationship for several SAE grade oils [7] 
In practice, it is common to describe the viscosity-temperature relationship of a lubricant in terms of its viscosity index (VI), a dimensionless value where the viscosity of a higher VI lubricant will not vary with temperature as much as a lower VI lubricant as shown in Figure 2.10. It is important to note that the viscosity index describes the magnitude of viscosity loss due to an increase in temperature, and not the value of kinematic viscosity at a particular temperature. It is possible for higher VI lubricants to have a lower viscosity than lower VI lubricants across a range of temperatures, and vice versa. Although the calculation of a lubricant's viscosity index is somewhat arbitrary in its definition, the process is universally accepted and well described by industrial standard ASTM D2270. [40].

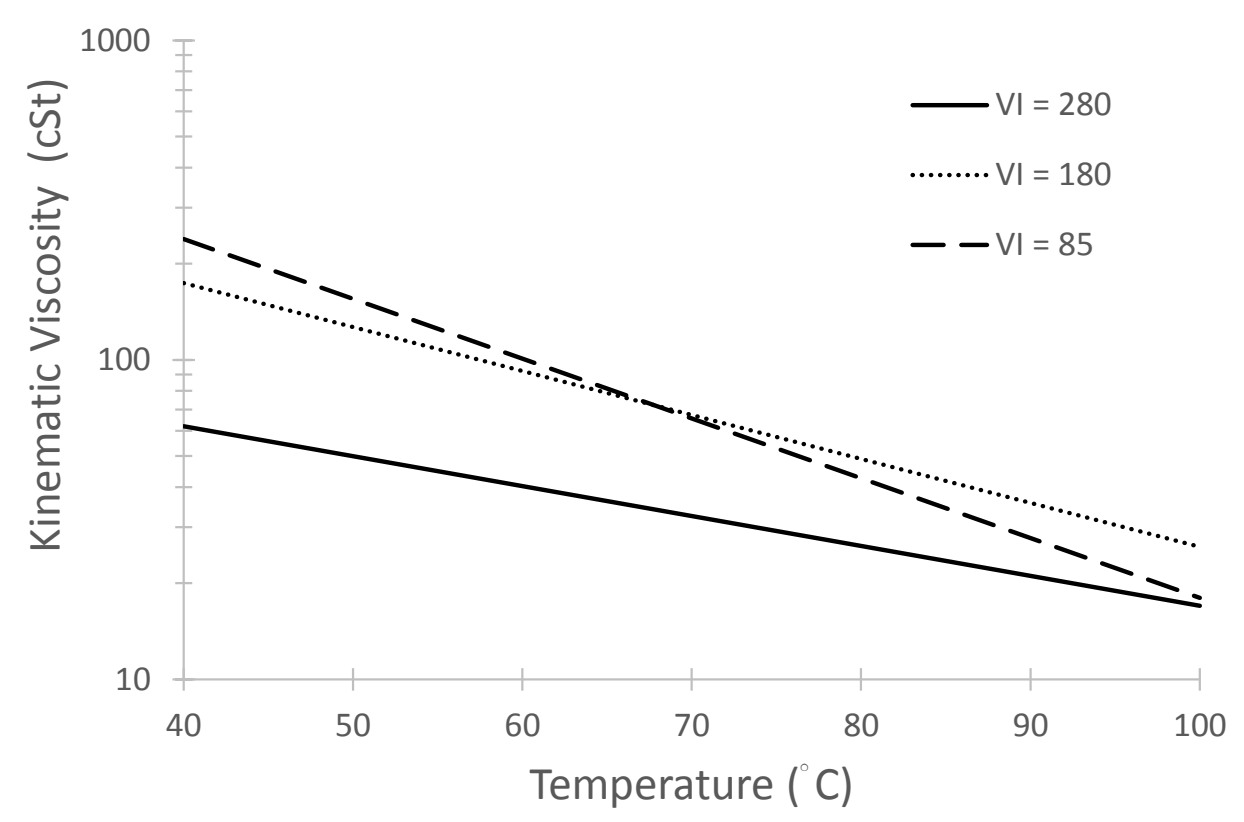

Figure 2.10: Viscosity-temperature relationship of different VI grade lubricants

\subsubsection{Viscosity Additives}

Where the decrease in lubricant viscosity across a range of temperatures is too great, or the base oil viscosity is too low to provide adequate film thickness at a specific 
temperature, viscosity index improvers (VIIs) may be added to a formulated lubricant to increase and stabilize the lubricant viscosity across a range of temperatures as shown in Figure 2.11. Viscosity index additives typically consist of high molecular weight oil-soluble polymers (on the order of 10'000 - 100'000 g/mol [41]) which are dissolved in the base oil to increase the viscosity index [5]. Viscosity index additives are commonly blended with a base oil in weight-percent concentrations from $0.8 \%$ to $2 \%[7]$.

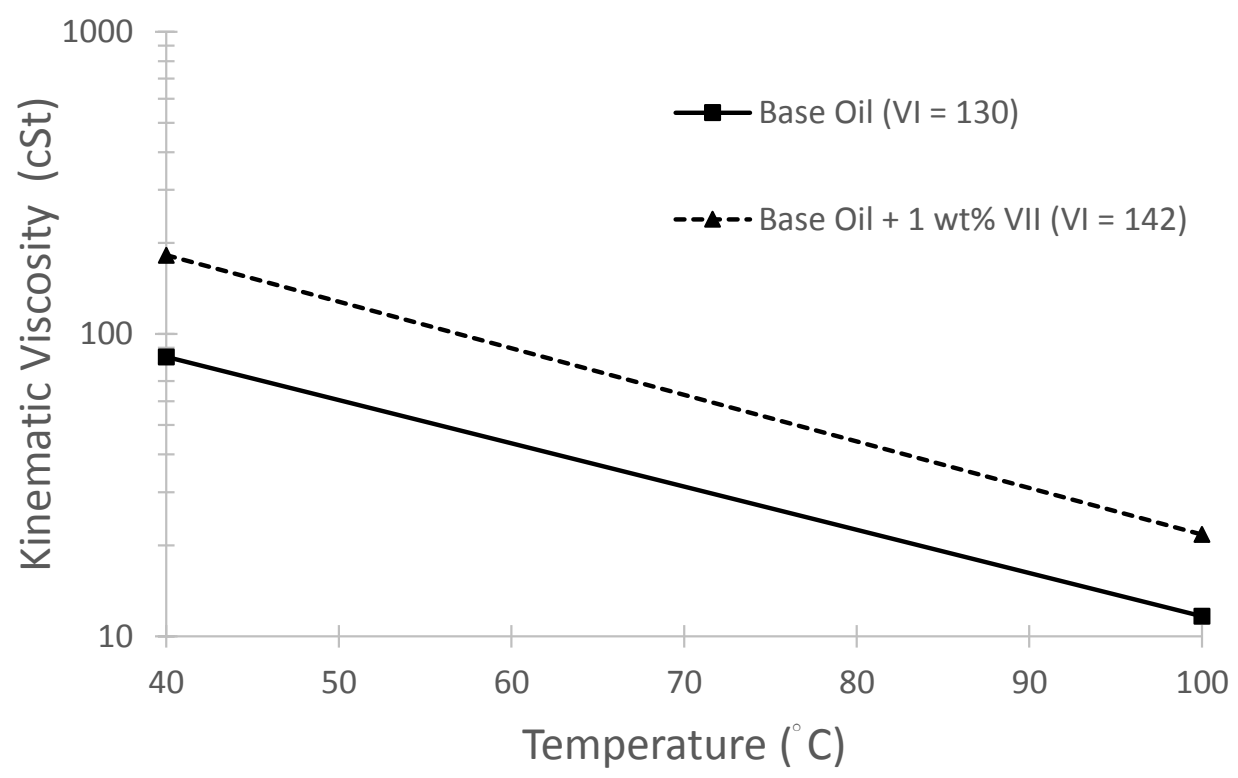

Figure 2.11: The addition of high molecular weight polymers can improve the viscosity-temperature performance of blended lubricants

It was originally believed that the mechanism of action of a viscosity index additive involved the hydrodynamic volume of the polymer molecule. It was noticed that the percent increase of lubricant viscosity due to polymer addition was greater at higher temperatures, which supported the claim that an increase in temperature caused an expansion of the polymer molecule and a correspondingly greater increase in lubricant viscosity at high temperatures [42]. However, with an increase in 
temperature, the polymer coil dimensions for certain types of VII can actually decrease as inferred by reduced viscosity measurements on dilute polymer solutions as shown in Figure 2.12 [7]. It is now theorized that the addition of polymer additives to a base oil solvent changes the entropy of activation of viscous flow for a dilute solution such that there is an increased resistance to fluid motion [43].

(a)

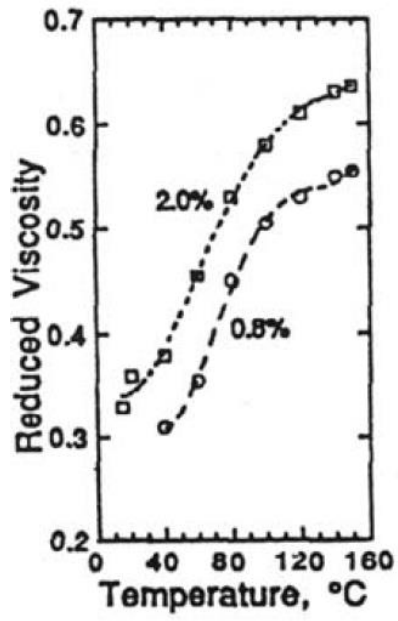

(b)

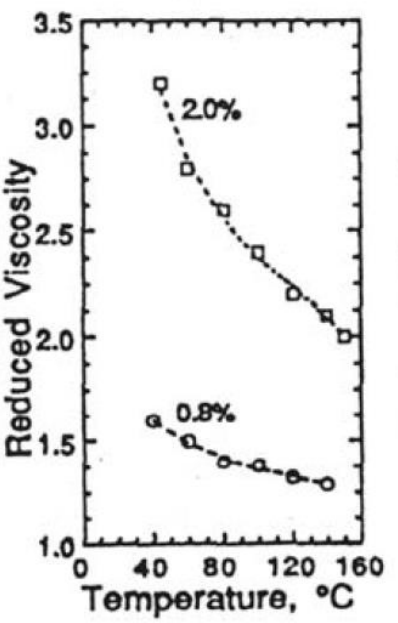

(c)

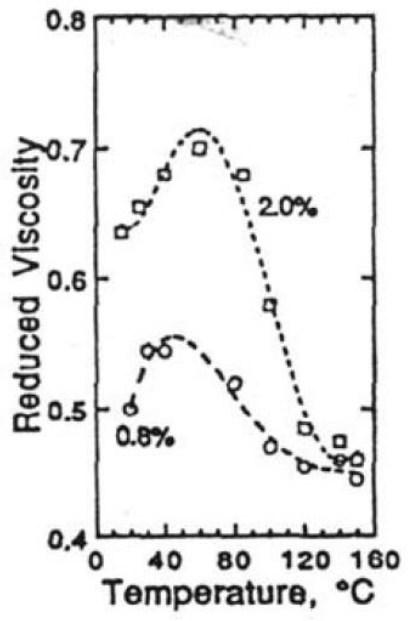

Figure 2.12: The polymer coil dimensions may increase or decrease with variations in temperature, as measured by changes in the reduced viscosity of a polymer solution containing (a) polyalkylmethacrylate, (b) ethylene-propylene copolymer, and (c) styrene-butadiene copolymer [7].

Polymers commonly used in petroleum-based lubricants may be added to a vegetable oil base to modify its viscosity properties. The tribological properties of EVA and SBS copolymers, which have seen use in petroleum-based lubricants, have been studied and proven to provide satisfactory viscosity improvement of a high-oleic sunflower oil [37]. These polymers are generally considered to be inert - not toxic or hazardous but also not biodegradable [44]. 
In other studies, ethyl cellulose polymers (which are non-toxic and commonly used in pharmaceuticals [45]) have been used as viscosity index additives in formulated vegetable oil lubricants, or as thickening agents in vegetable oil-based greases. High-oleic sunflower oil and castor oil based lubricants blended with ethyl cellulose polymers were shown to have a higher viscosity and viscosity index, greater lubricity and film thickness across a wide range of temperatures, and a lower pour point than the stock base oil [3] [32].

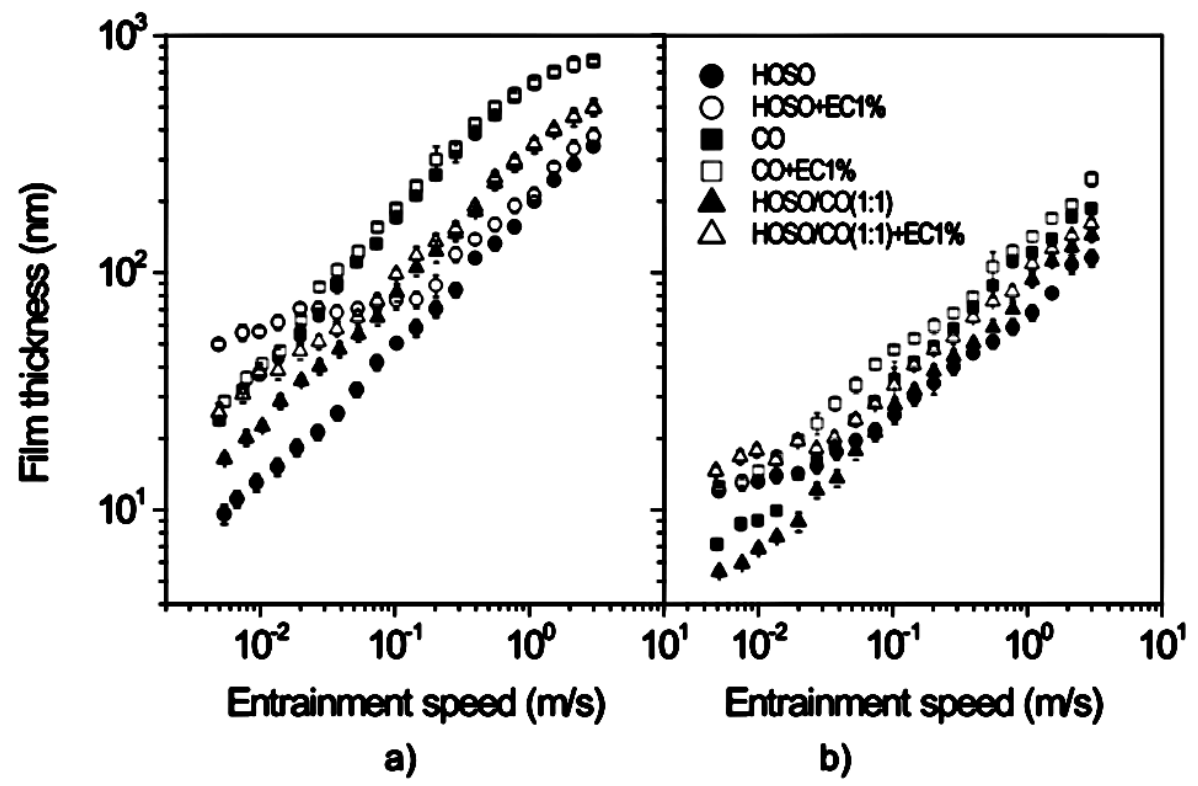

Figure 2.13: The addition of an ethyl cellulose polymer increased the film thickness compared to the base oil. Measured at (a) $40^{\circ} \mathrm{C}$ and (b) $100^{\circ} \mathrm{C}$ [3]. 
Table 2.4: The addition of an ethyl cellulose polymer increased the viscosity and viscosity index compared to the base oil [3]

\begin{tabular}{rc|ccc} 
Base Oil & $\mathrm{c}($ wt. $\%)$ & $\nu_{40}{ }^{\circ} \mathrm{C}$ & $\nu_{100}{ }^{\circ} \mathrm{C}$ & $\mathrm{VI}$ \\
\hline Castor Oil & 0.0 & 242 & 21 & 116 \\
& 0.5 & 420 & 31 & 104 \\
& 1.0 & 547 & 40 & 117 \\
& 2.0 & 686 & 69 & 176 \\
\hline High-oleic sunflower oil & 0.0 & 39 & 10 & 257 \\
& 1.0 & 51 & 15 & 314 \\
& 2.0 & 59 & 19 & 342
\end{tabular}

\subsubsection{Solution Properties}

Blended polymer and base oil solutions behave as a dilute solution [8]. Aside from measurements of kinematic viscosity of a formulated lubricant, there are several other measurements which have been developed from the field of dilute polymer viscometry to describe the behaviour of viscosity additive polymers in a base oil solution. These equations are summarized in Table 2.5.

Table 2.5: Viscometric terminology

\begin{tabular}{c|cc}
\multicolumn{1}{c}{ Name } & Definition & Symbol \\
\hline Relative viscosity & $\eta / \eta_{0}$ & $\eta_{\text {rel }}$ \\
Specific viscosity & $\eta_{\text {rel }}-1$ & $\eta_{s p}$ \\
Reduced viscosity & $\eta_{s p} / c$ & $\eta_{\text {red }}$ \\
Inherent viscosity & $\ln \left(\eta_{s p} / c\right)$ & $\eta_{i n h}$ \\
Intrinsic viscosity & $\lim \left(\eta_{s p} / c\right)_{c \rightarrow 0}$ & {$[\eta]$}
\end{tabular}

Solutions of polymers in a base oil may also be described by the well known Huggins 
(Equation 2.4) and Kraemer (Equation 2.5) equations [8].

$$
\begin{gathered}
\eta_{s p}=[\eta] c+k_{h}[\eta]^{2} c^{2} \\
\ln \left(\eta_{\text {rel }}\right)=[\eta] c+k_{k}[\eta]^{2} c^{2}
\end{gathered}
$$

Where the Huggins and Kraemer constants ( $k_{h}$ and $k_{k}$, respectively) are determined experimentally and contain information about interactions between the polymer coils and the base oil in solution [46]

The Huggins and Kraemer equations may be used in combination with experimental viscosity measurements to determine the intrinsic viscosity $[\eta]$ of a polymer solution. The intrinsic viscosity is a measure to quantify the viscosity contribution of polymer molecules at a theoretically infinite dilution, where individual polymer molecules interact with the solvent to increase the viscosity of the solution without the influence of macromolecular forces from other polymer molecules. It cannot be determined through direct means, and must be extrapolated from a series of viscosity measurements. Although it is not possible to make comparisons between different additive chemistries, the intrinsic viscosity is useful in determining the effectiveness of different molecular weight polymers within the same type of additive [8].

\subsubsection{Permanent Viscosity Loss due to Mechanical Shear}

When lubricants containing polymer additives are subject to high rates of shear in machinery, the bond strengths which hold the polymer molecule together may be exceeded and the polymer molecule will break down into two fragments of 
shorter chain length. The decrease of polymer chain length leads to a permanent degradation and loss of lubricant viscosity and a decrease in the viscosity index, as the shortened polymer chains are less effective at performing the function of a viscosity index additive [14]. The process is demonstrated in Figure 2.14 [8].

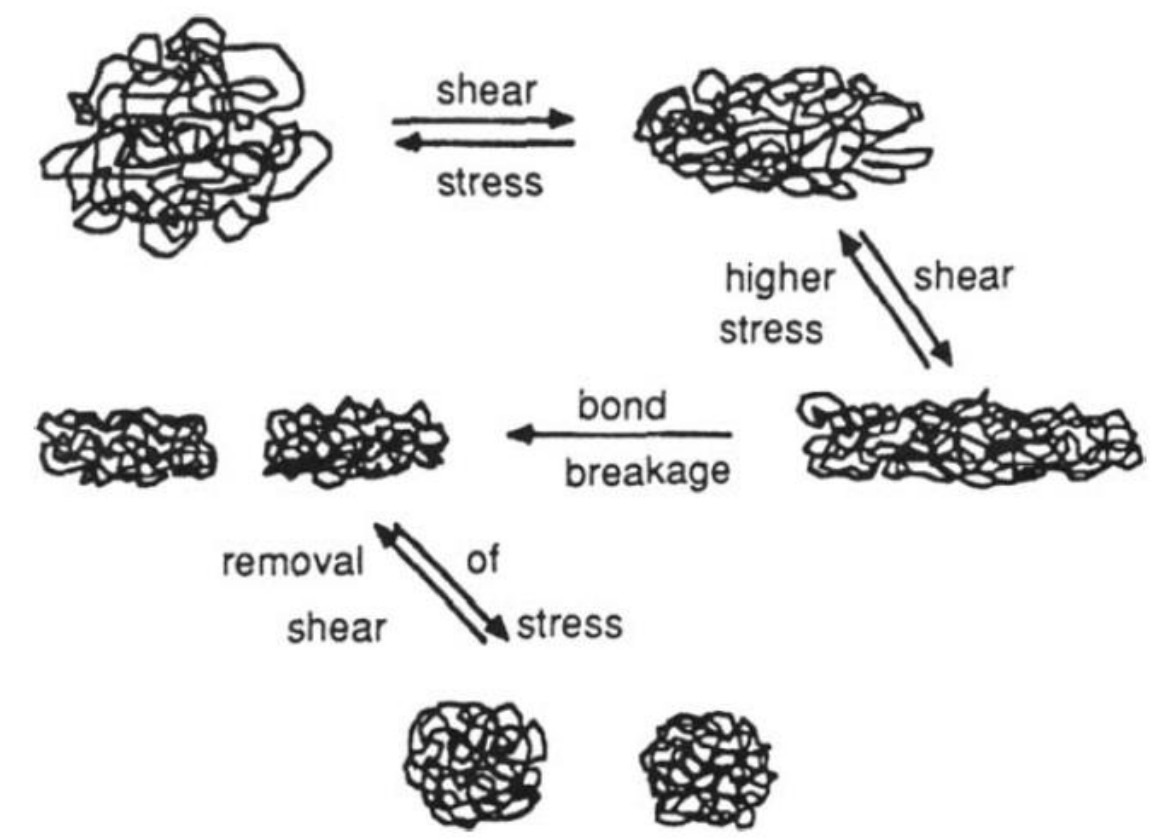

Figure 2.14: Schematic representation of the permanent degradation of a polymer molecule at high rates of shear [8]

The higher the polymer molecular weight, the more likely the lubricant will experience permanent viscosity loss due to mechanical shear [47]. This mechanical degradation process is self limiting. After a number of shearing cycles, a molecular weight is reached such that the energy concentration during polymer coil distortion is not enough to break the bonds in the polymer chain. Shear degradation is generally characterized by a rapid decrease in lubricant viscosity as the larger molecules are broken down first, followed by a gradual decrease towards a lower viscosity limit as the remaining smaller polymer molecules approach the limiting molecular weight for shear degradation [8]. 
The shear degradation of polymer lubricants may be described by the percent change of permanent viscosity loss (PVL) with shear as described by Equation 2.6

$$
P V L=\frac{\eta_{\text {unsheared }}-\eta_{\text {sheared }}}{\eta_{\text {unsheared }}} \cdot 100
$$

As lubricants may be blended for a wide range of viscosities, it can be more useful to characterize the permanent viscosity loss in terms of its shear stability index (SSI), which describes the percentage of polymer-contributed viscosity which is lost due to mechanical shear. Polymer additives which experience greater shear degradation will have a higher shear stability index than polymers which experience a lesser degree of shear degradation.

$$
S S I=\frac{\eta_{\text {unsheared }}-\eta_{\text {sheared }}}{\eta_{\text {unsheared }}-\eta_{0}} \cdot 100
$$

It is also acceptable to characterize the degree of shear degradation by measuring the change in the lubricant's intrinsic viscosity. This method is useful for describing changes in the ability for a polymer molecule to act as a thickening agent and a viscosity index additive [48].

\subsection{Experimental Methods in Mechanical Degra- dation of Polymers}

The most comprehensive method for determining the shear stability of a lubricant is to test it in the equipment or machinery in which the lubricant is designed for [9]. However, this can be an expensive and time consuming endeavour. As such, a variety 
of experiments have evolved over the years to accelerate the rate of degradation to characterize the degree of permanent viscosity loss due to shear. However, each method is not without some controversy as to the effectiveness of correlating laboratory results with in-service performance [49] [9].

The most widely used shear simulator involves pumping the sample lubricant through a diesel fuel injector nozzle to generate significant rates of shear which are capable of breaking the polymer bonds [14] [9]. The procedure is well accepted by industry and researchers, and described by ASTM6278 [50].

A separate method for assessing the shear stability of lubricants involves ultrasonic irradiation of the sample fluid. This test produces microscopic cavitation bubbles which quickly collapse and create localized shear fields which are strong enough to break the polymer molecules. The test procedure has also been developed into an industrial standard ASTM2603 [51].

As each shear simulator quantifies the degree of shearing differently (ASTM6278 measures viscosity loss with the number of shear cycles through the diesel fuel injector, and ASTM2603 measures viscosity loss with the amount time a sample is exposed to ultrasonic waves), debate surrounding the effectiveness of shear stability simulators has focussed around variations in the limiting viscosity achieved by each test method [52] [53] as shown in Figure 2.15. 

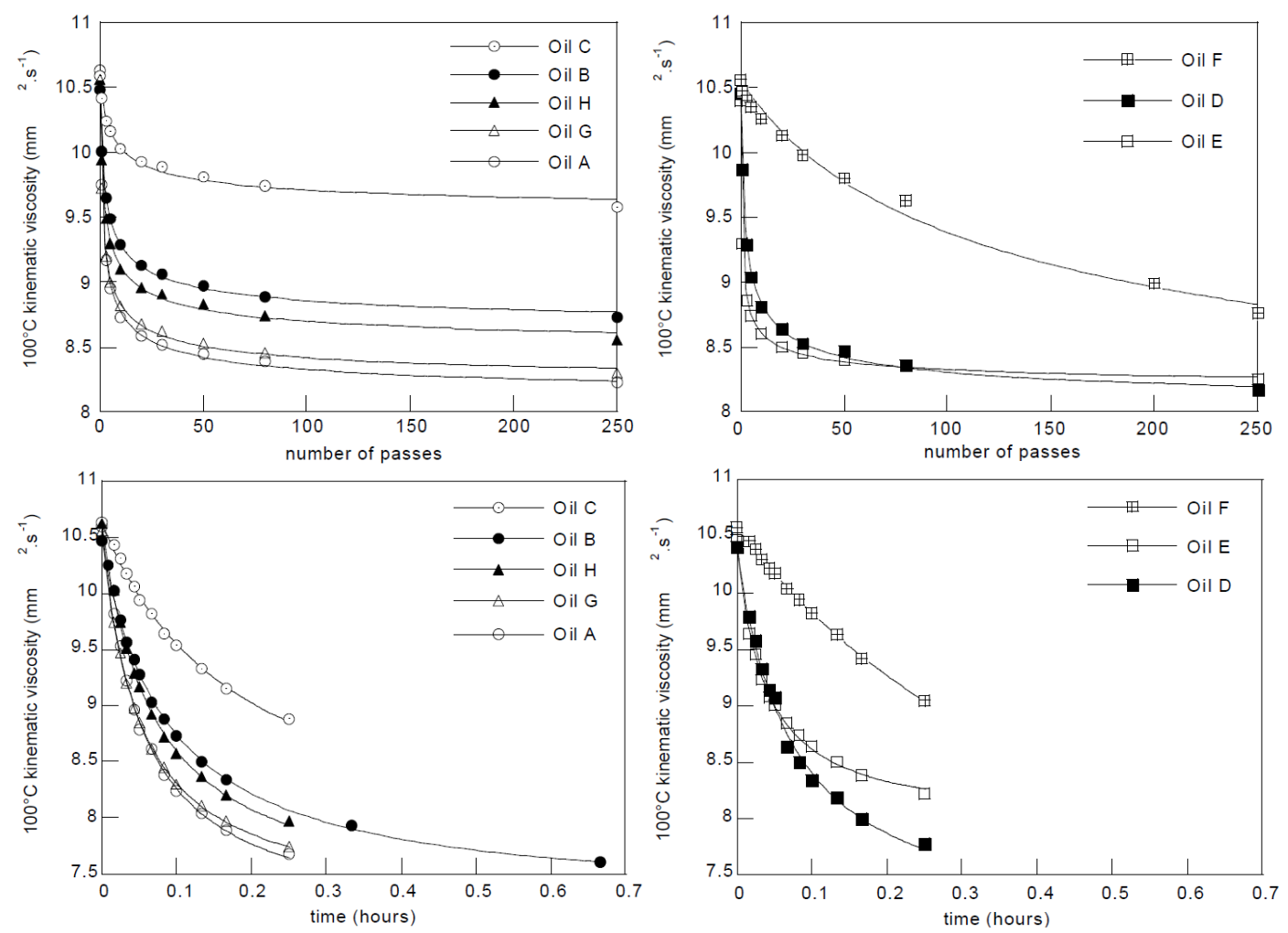

Figure 2.15: Changes in the kinematic viscosity measured at $100^{\circ} \mathrm{C}$ for lubricant blends sheared with the diesel injector test (top row) and the sonic shear stability test (bottom row) [9]

It should be noted that each of these tests were developed to test the shear stability of polymer additives which are commonly used for petroleum-based lubricants. It is unclear whether there is a significant bias in the test results when evaluating the shear stability of biodegradable polymer additives which have yet to be commonly adopted by industry. While surveying literature, only a single example was found which examined the shear stability of biodegradable lubricants using a diesel fuel injector test [54]. 


\section{Chapter 3}

\section{Experimental Setup}

The objective of this research was to test the shear stability of a biodegradable vegetable oil lubricant which was blended with a viscosity index additive. While it is always desirable to test a formulated lubricant in-situ [9], the diesel fuel injector experiment is an acceptable means of quantifying the shear stability of polymer containing lubricants as described by ASTM6278 [50]. A previous colleague had also developed a functioning diesel fuel injector apparatus for shearing samples of heavily oxidized mineral oil [55], and it was desirable to re-purpose equipment where possible.

\subsection{Materials}

High-oleic sunflower oil and castor oil used in this study were commercially available with a fatty acid composition as described in Table 3.1. High-oleic sunflower oil was purchased from Jedwards International, and the castor oil was purchased from Sigma Aldrich Canada. Oils were used as received without any further purification or chemical modification. Ethyl cellulose with a $48 \%$ ethoxy content was purchased from Sigma Aldrich Canada. Polymers of this type are typically sold based on their dynamic viscosity in a toluene and ethylene solution. The polymer molecular weights were determined in previous work of Delgado et al. [3] as summarized in Table 3.2. 
Table 3.1: Fatty acid composition (as provided by manufacturer data sheets) of the vegetable oils used in this research.

\begin{tabular}{r|cc} 
& \multicolumn{2}{|c}{ Fatty acid content (wt. \%) } \\
Fatty acid type & Castor Oil & High-oleic sunflower Oil \\
\hline Palmitic (C16:0) & - & 4.6 \\
Stearic (C18:0) & - & 3.0 \\
Oleic (C18:1) & 7 & 80.1 \\
Ricinoleic (C18:1: OH) & 87 & - \\
Linoleic (C18:2) & 3 & 10.3 \\
Linolenic (C18:3) & - & 0.1
\end{tabular}

Table 3.2: Physical properties of polymer additives

\begin{tabular}{r|cc} 
& Ethyl cellulose $(45 \mathrm{cP})$ & Ethyl cellulose $(100 \mathrm{cP})$ \\
\hline Physical form & white granules & white granules \\
Molecular weight $(\mathrm{g} / \mathrm{mol})[3]$ & 68960 & 76530
\end{tabular}

\subsection{Preparation of Lubricant Blends}

A base oil blend was prepared in batches with equal-weight proportions of high-oleic sunflower oil and castor oil in a $500 \mathrm{ml}$ beaker. An Intell-Lab PBX-3200 was used to obtain a weight fraction of solution to within $\pm 0.01 \mathrm{~g}$. The blended base oil was heated to $50^{\circ} \mathrm{C}$ using a hot plate with low speed magnetic stirring for 
approximately 10 minutes to ensure adequate mixing. The oil temperature was measured independently using a type $\mathrm{J}$ thermocouple immersed in the oil.

A Sartorius ENTRIS64-1S analytical balance with an accuracy of $\pm 0.1 \mathrm{mg}$ was used to portion out a desired amount of solid polymer on a tared filter paper. In previous studies with ethyl cellulose polymers in a vegetable oil, a $1 \%$ wt. concentration of polymer additive provided adequate thickening and it was decided to use this as a baseline for formulated lubricants in this experiment [32] [34] [3]. A weighed sample of the blended base oil was heated up to $150^{\circ} \mathrm{C}$ and the polymer powder was slowly added while stirring with a magnetic stir bar at low speed. The sample was held at a constant temperature with stirring for approximately 60 minutes until there were no visible solid particles. However, it should be noted that the solution was still observed to have a cloudy or hazy appearance at this point. The sample temperature was then incrementally lowered to room temperature over the course of approximately 90 minutes with intermittent stirring. At this point, the sample appeared homogeneous with no visible precipitate or cloudiness. Viscometric properties of the base oils and blended lubricants are summarized in Table 3.3.

Table 3.3: Properties of base oils and formulated lubricants with $1 \%$ wt. ethyl cellulose polymer additives. EC45 denotes a lubricant blend with ethyl cellulose (45 cP) and EC100 denotes a lubricant blend with ethyl cellulose (100 cP)

\begin{tabular}{c|ccccc} 
Sample & HOSO & CO & HOSO $/$ CO $(1: 1)$ & EC45 & EC100 \\
\hline$\nu_{40^{\circ} \mathrm{C}}(\mathrm{cSt})$ & 38.81 & 239.0 & 84.07 & 184.2 & 217.6 \\
$\nu_{100^{\circ} \mathrm{C}}(\mathrm{cSt})$ & 8.41 & 18.51 & 11.65 & 21.87 & 25.8 \\
VI & 201 & 85 & 130 & 142 & 151
\end{tabular}




\subsection{Shearing Apparatus}

A diesel fuel injector apparatus was constructed previously to examine the effects of combined oxidation and shear on mineral oil samples [55]. Although it may be difficult to correlate the permanent viscosity loss from shearing through a diesel fuel injector to any specific application, literature indicates that a diesel fuel injector is still widely used for shear degradation of lubricating oils and other polymer-containing fluids [9]. The concept of a diesel fuel injector shear apparatus is also supported by ASTM 6278 [50].

The apparatus was originally re-purposed from a diesel fuel Ignition Quality Tester $\left(\mathrm{IQT}^{T M}\right)$ that had previously been used in combustion research. Previous efforts by LaForge [55] converted the IQT into an apparatus which could shear samples of oil one cycle at a time, meaning the volume of sample had to be collected and re-loaded after each cycle. For the purpose of this experiment, modifications were completed to allow for a continuous circulation of sample fluid and to improve the consistency of air pressure delivered to the pneumatic actuator as described below.

The stock injector nozzle was replaced with a Bosch type DN8S2 as per ASTM 6278 specifications. Injections were triggered by an Arduino MEGA2560 microcontroller board which commanded the actuation of an ASCO 8316G14 solenoid valve. Fluid injection was achieved with a pneumatic actuator assembly (shown in Figure 3.1) which was supplied high pressure air from a pair of Haskel HAA31-2.5 air pressure amplifiers operating in parallel to increase the available flow rate of high pressure air. In order to ensure a consistent supply of pressure for each injection, the apparatus was modified so that a primary accumulator tank was charged to approximately 250 psig by the air pressure amplifiers, and the air actuator was supplied by a pressure 
regulated secondary accumulator which was charged to approximately 150 psig. The secondary accumulator tank pressure was also monitored with a PX-309 pressure transducer which functioned as a pressure switch to ensure that no injections would occur unless the secondary accumulator was sufficiently pressurized. A schematic of the modified injector assembly is shown in Figure 3.2

Oil was injected into a collector chambre with a hemispherical cavity which was also filled with sample fluid to reduce air entrapment and minimize the risk of oxidation [55]. From the atomization chamber, oil flowed freely back into the reservoir to allow for a continuous circulation of sample fluid. In this manner, the injector served as a constant displacement pump which circulated the sample fluid for the required number of shear cycles. The reservoir was pressurized to $60 \mathrm{psig}$ in order to force fluid into the injector pump barrel cavity in between injections. Sample fluid is separated from pressurized air in the reservoir by a sealed PTFE plunger. 


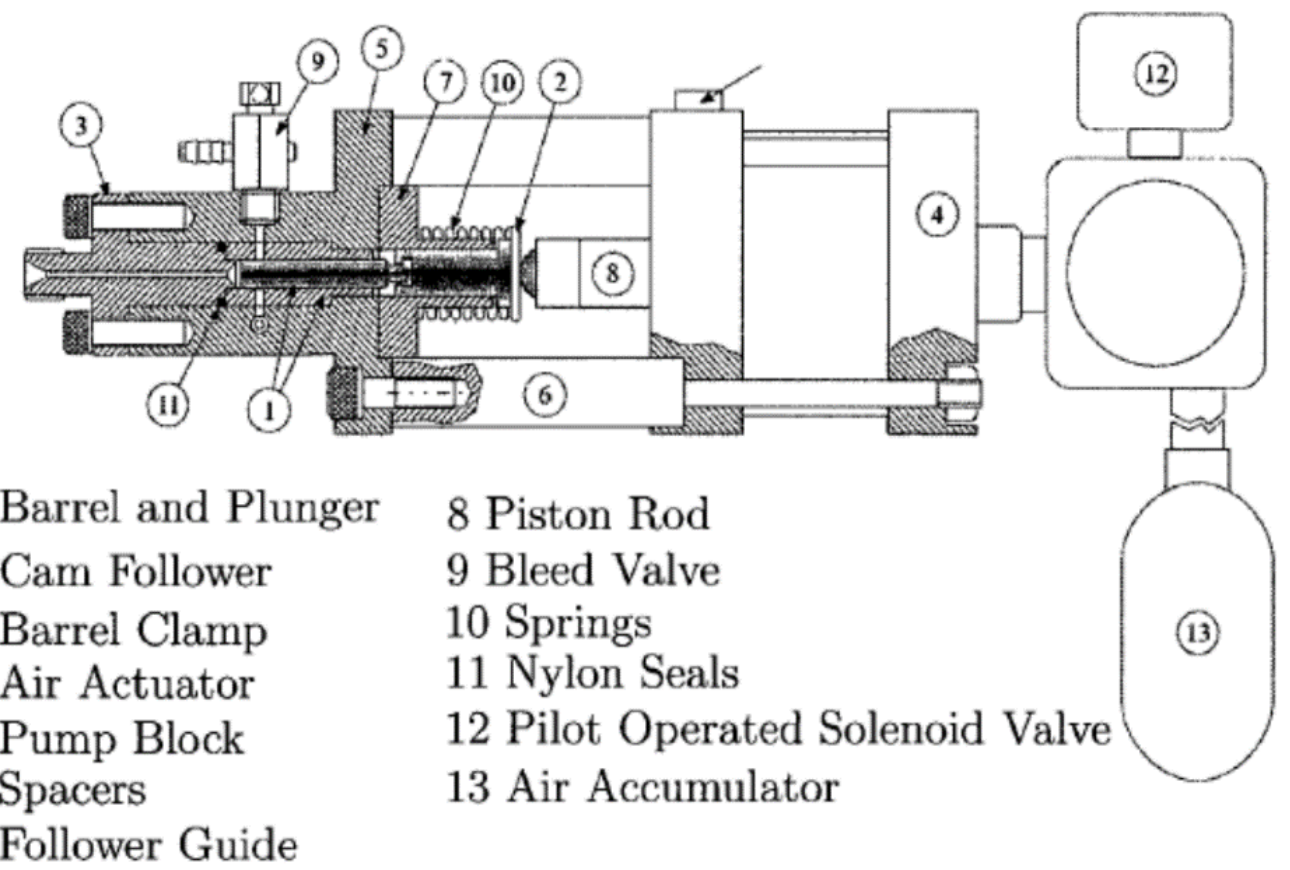

Figure 3.1: Details of the injector pump body [10]. 


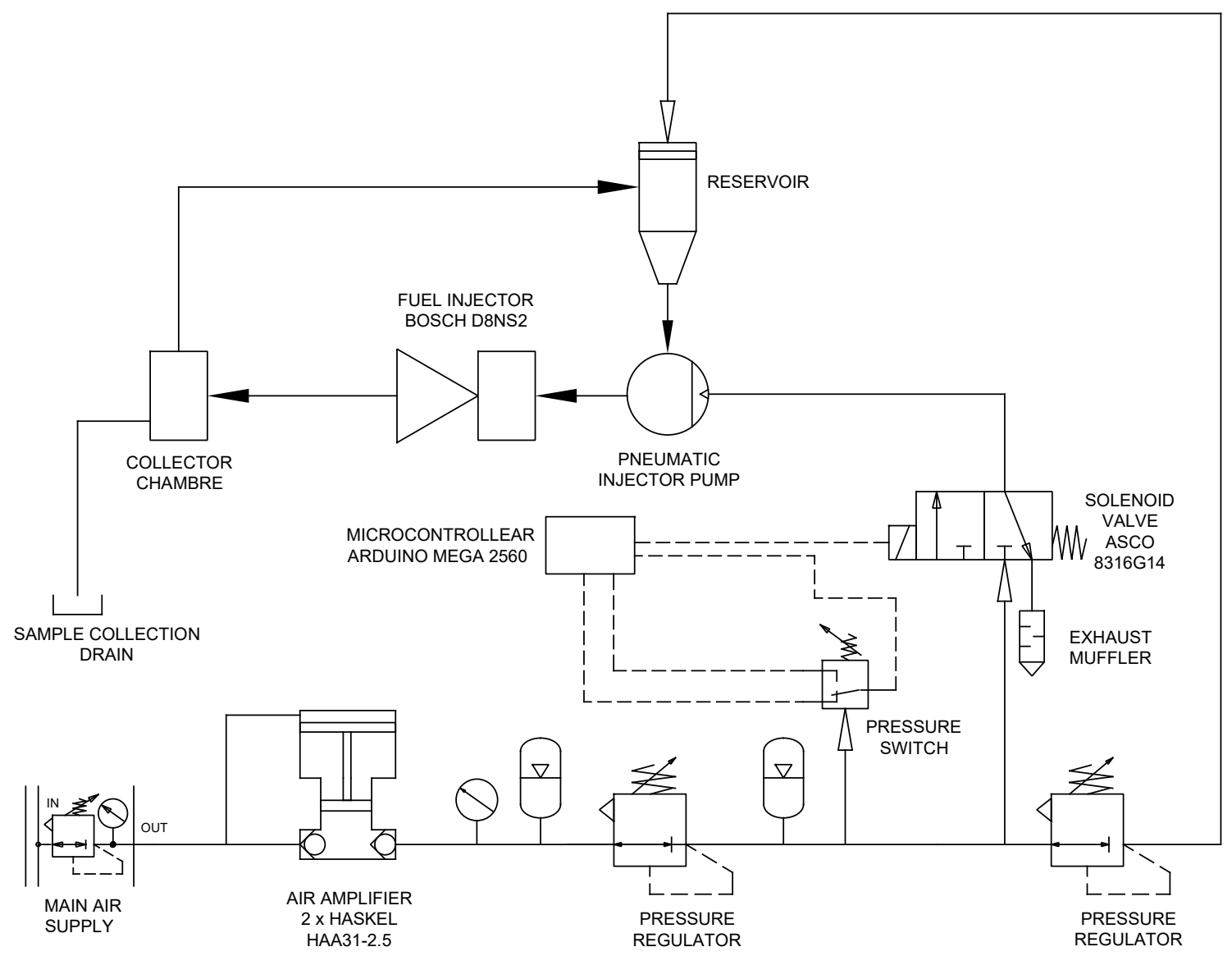

Figure 3.2: Schematic of the modified injector assembly. White arrows indicate the pneumatic subassembly and solid arrows indicate the flow of sample oil through the apparatus 


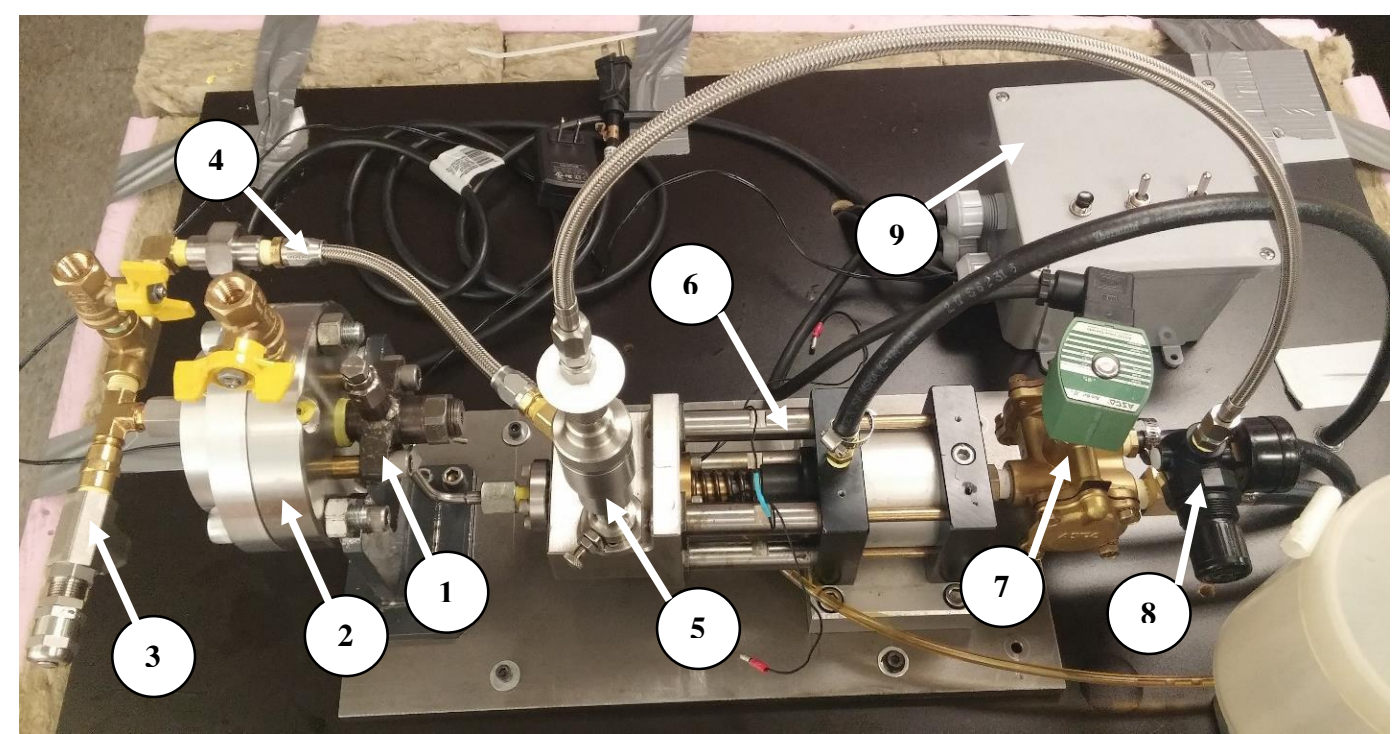

1. Diesel fuel injector holder

2. Collector chambre

3. Pressure relief valve

4. Sample fluid return line

5. Reservoir
6. Pneumatic actuator

7. Solenoid valve

8. Reservoir pressure regulator

9. Microcontroller enclosure

Figure 3.3: Photo of the modified injector assembly with key components labelled.

A $60 \mathrm{ml}$ sample of blended lubricant to be sheared was poured into the reservoir and a vented opening at the top of the collector chambre. The fluid was allowed to settle for approximately 10 minutes before closing the vent on the collector chambre and capping the reservoir. The main air supply was switched on and the reservoir was pressurized to 60 psig before arming the microcontroller to start the injection process. After 30 cycles of continuous shearing (approximately 15000 injections), the system was de-pressurized and $10 \mathrm{ml}$ of fluid was drained and discarded from the drain valve at the bottom of the atomization chambre. Approximately $5 \mathrm{ml}$ of 
fluid was also pipetted and discarded from the top of the reservoir. The remaining volume of sheared fluid was collected in a clean sample vial for determination of kinematic viscosity. The reservoir, collector chambre, and return lines were also partially disassembled in between tests and cleaned with successive rinses of toluene and acetone to remove any residual lubricant. As an additional measure to reduce possible contamination, approximately $20 \mathrm{ml}$ of the subsequent sample to be tested was pumped through the fuel injector to flush out any remaining lubricant from the previous test before being discarded.

As per ASTM 6278 standards, a calibration oil (RL233) was cycled through the shearing apparatus 30 times in order to verify that the fuel injector was creating a consistent and predictable amount of mechanical degradation. The nozzle pressure was adjusted until the permanent viscosity loss at $100^{\circ} \mathrm{C}$ as measured by capillary viscometer was measured to be between 2.7 - 2.9 cSt [50]. Several calibration tests were also completed (30 SC-E and $30 \mathrm{SC}-\mathrm{F}$ ) in between shear tests of the blended lubricant to ensure that the shearing process remained consistent. Calibration test results are shown in Table 4.1 .

\subsection{Viscosity Measurements}

As previously discussed, the viscosity of a lubricant is one of the most important physical properties. Measurements of kinematic viscosity were used to characterize the effects of shear as described in the following sections. 


\subsubsection{Determination of Kinematic Viscosity}

Kinematic viscosity measurements were completed as outlined in ASTM standard D445-15 [11] with the exception that viscometers were cleaned with three successive rinses of toluene and acetone instead of chromic acid as recommended. Chromic acid can cause severe health side effects and requires extreme caution to use correctly. As the viscometers were purchased new, and the duration of testing was relatively short, the risk of sample contamination from varnish or surface deposits was low compared to the potential risks of using chromic acid to clean the viscometers.

All kinematic viscosity measurements were performed using calibrated CannonUbbelohde capillary tube viscometers ranging from size 100 to 300 . A capillary viscometer functions by measuring the time required to drain a sample reservoir of defined volume under the force of gravity. Cannon-Ubbelohde viscometers were preferred as the viscometer constant is the same for all temperatures and they require no kinetic energy corrections within a specified range of viscosities. The viscometer was immersed in a constant-temperature oil bath conforming to ASTM D445 (shown in Figure 3.4 ) with a precision of $\pm 0.1^{\circ} \mathrm{C}$ provided by a Chemglass CG-15001-30 digital PID temperature controller. Instructions for use of the Cannon-Ubbelohde viscometer are available from Cannon Instruments [56]. 


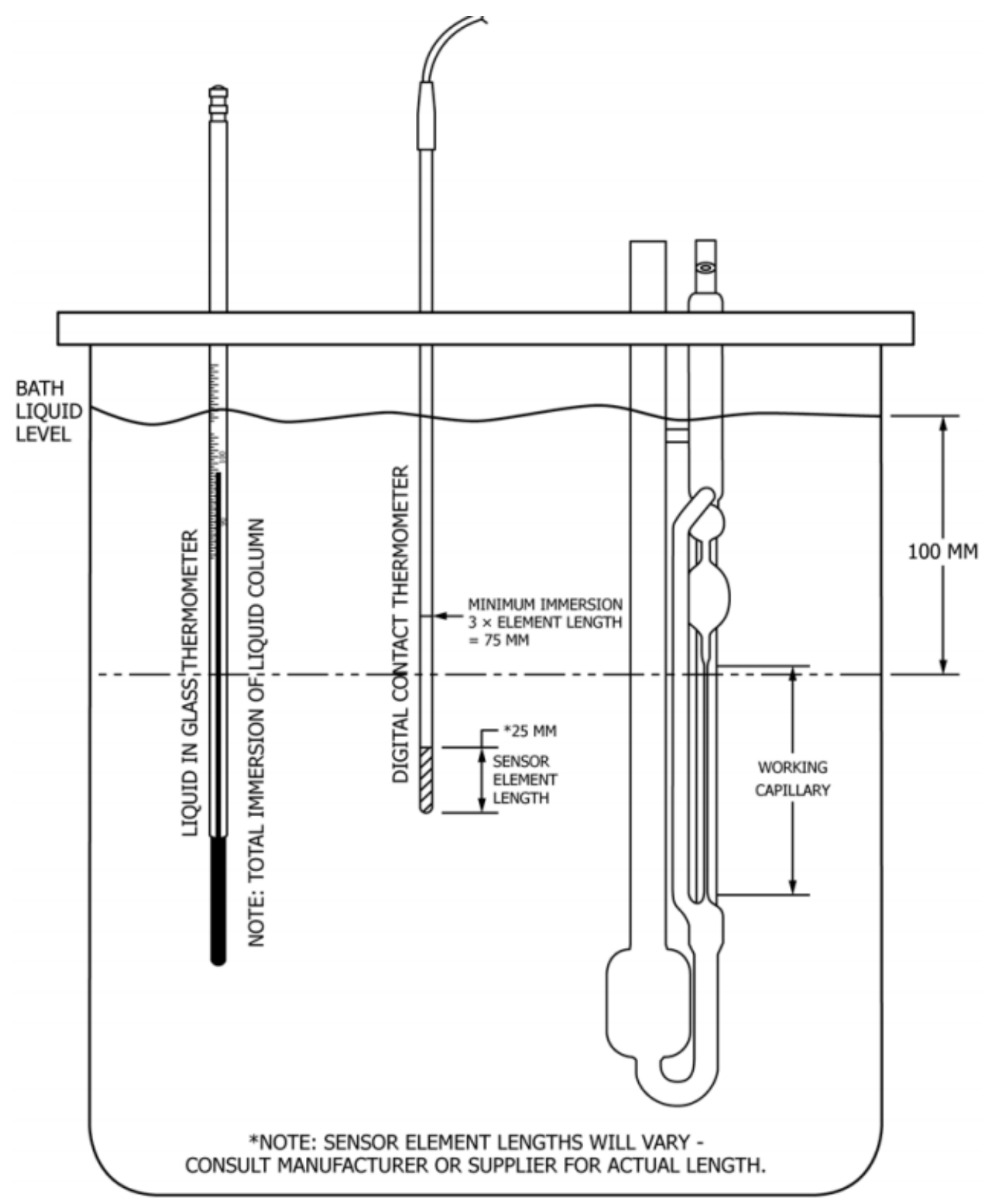

Figure 3.4: Schematic of the oil bath as specified by ASTMD445 [11] 


\subsubsection{Determination of Intrinsic Viscosity}

Intrinsic viscosity measurements were used to characterize the effectiveness of the polymer additive to perform as a thickening agent and viscosity index additive. The determination of intrinsic viscosity required measurements of the specific and inherent viscosity (which can be determined from kinematic viscosity measurements as described in Section 2.3.3) at increasingly dilute polymer concentrations starting from the $1 \%$ wt prepared lubricant blend. Unsheared and sheared samples were progressively diluted with base oil to $0.75 \%$ wt, $0.50 \%$ wt, and $0.25 \%$ wt concentrations of polymer additive in base oil to construct a plot similar to that as shown in Figure 3.5. From these plots, the intrinsic viscosity was calculated as the y-intercept of a linear regression of the measured data. The procedure is well documented as an industrial standard in ASTM2857 [12].

As a comparison, a simple MATLAB script was constructed to fit a least-squares second order polynomial to the relative and inherent viscosity data to simultaneously solve for the intrinsic viscosity as well as the Huggins and Kraemer coefficients which appear in Equations 2.4 and 2.5. The sample code is included in Appendix B for reference. 


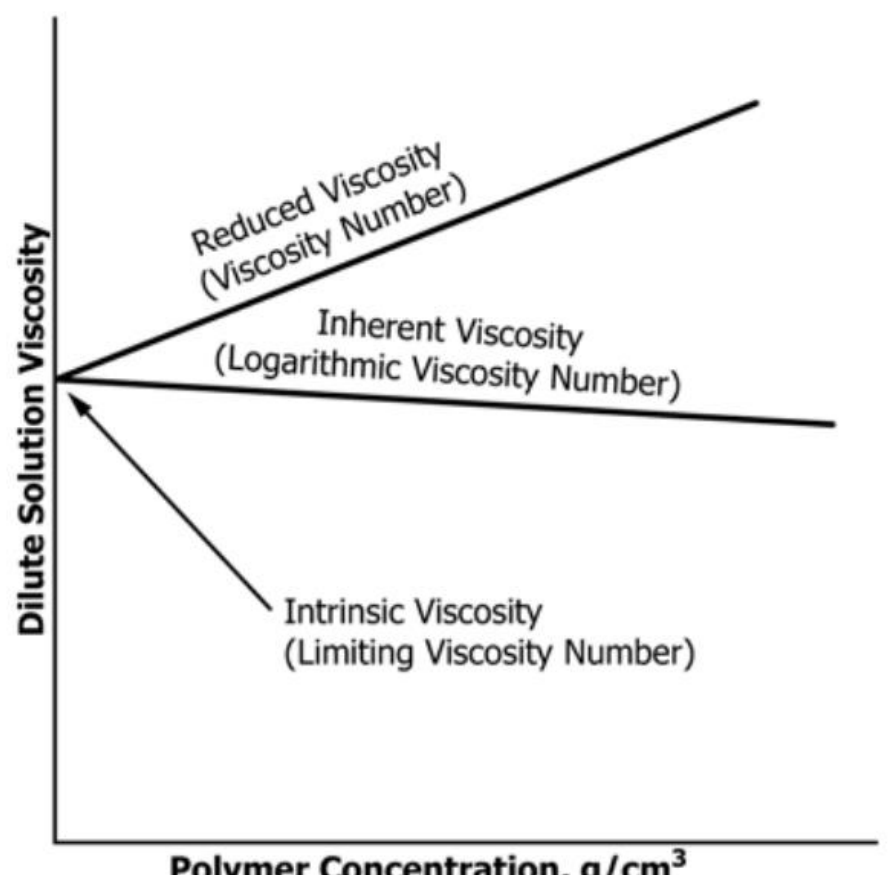

Polymer Concentration, $\mathrm{g} / \mathrm{cm}^{3}$

Figure 3.5: Example plot constructed to determine the intrinsic viscosity of polymer containing solutions [12]

\subsection{Determination of Solution Density}

The density of base oils and formulated lubricants was determined at $40^{\circ} \mathrm{C}$ and $100^{\circ} \mathrm{C}$ using calibrated Gay-Lussac density bottles (shown in Figure 3.6) which were purchased from Sigma Aldrich. Density bottles were manufactured in accordance with DIN ISO 3507 and are calibrated by the manufacturer. The procedure may be described as follows.

1. Heat the oil bath to the desired temperature and allow approximately 30 minutes for the temperature to stabilize.

2. The inner and outer surfaces of the density bottle were cleaned with three 
successive rinses of toluene. A final rinse of acetone was used to remove any trace of toluene. When handling the bottle by hand, a fresh pair of latex or neoprene examination gloves were worn to avoid contaminating the outer surface with oils or dirt from skin contact.

3. The empty weight of the bottle and stopper was measured. In this experiment, a Sartorius ENTRIS64-1S with an accuracy of $\pm 0.1 \mathrm{mg}$ was used to determine the weight of the empty and filled density bottles.

4. With the stopper removed, a 10 gauge blunt-tip luer lock needle and syringe was used to fill the density bottle to approximately $1 / 3$ of the height of the neck, being careful not to allow bubbles to form in the oil.

5. In cases where visible bubbles formed in the oil, the bottle was placed in a small drying oven which was heated to $70^{\circ} \mathrm{C}$ to reduce the fluid viscosity such that bubbles would float to the surface and dissipate more easily. The bottle was allowed to cool back to room temperature for approximately 30 minutes before proceeding.

6. The stopper was placed into the bottle, matching the markings of the bottle and stopper. Some of the testing fluid will run up the capillary and escape out the top of the bottle. This is normal.

7. The bottle was positioned in a holder and immersed in the oil bath to a point just below the opening of the neck and allowed to come to temperature for approximately 30 minutes.

8. The top surface of the stopper was carefully cleaned with a lint-free tissue while avoiding contact with the capillary. In order to obtain accurate results, the testing liquid must be level with the upper end of the capillary. After cleaning, 
the bottle was observed for another 10 minutes to ensure that no further liquid seeped out of the capillary due to fluid expansion.

9. The bottle was removed from the oil bath holder and allowed to cool to room temperature.

10. The outside surface was carefully cleaned with acetone and lint-free tissues to remove any trace of bath oil. The bath oil was a mineral oil and completely soluble in acetone.

11. The final weight was measured using a Sartorius ENTRIS64-1S.

12. Fluid density was calculated as the difference between empty and filled weight divided by the calibrated fill volume provided on the calibration certificate.

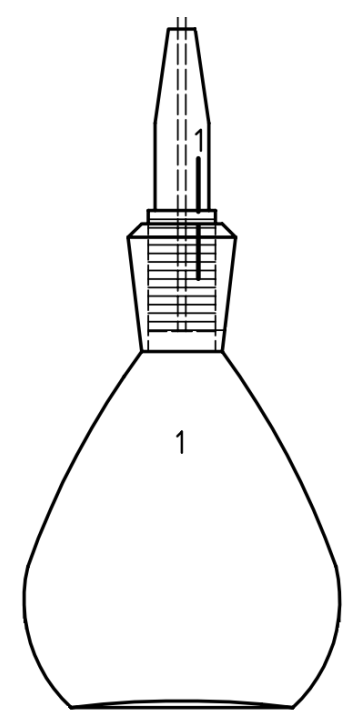

Figure 3.6: Calibrated Gay-Lussac density bottles were used for determination of fluid density at $40^{\circ} \mathrm{C}$ and $100^{\circ} \mathrm{C}$ 


\section{Chapter 4}

\section{Experimental Results}

As described in Chapter 3, a diesel fuel injector apparatus was commissioned to induce mechanical degradation of a high-oleic sunflower oil and castor oil blend with $1 \%$ wt. concentration of ethyl cellulose polymer additive. Cannon-Ubbelohde viscometers were used to measure the kinematic viscosity of prepared solutions before and after shearing. Further viscometric properties were determined for the sheared and unsheared samples to characterize the shear degradation for two molecular weights of ethyl cellulose.

\subsection{Calibration with RL233 Reference Oil}

As the diesel injector apparatus used in this experiment was re-purposed from existing equipment and not supplied as a complete shear stability tester, it was necessary to adjust the nozzle pressure to produce a comparable amount of mechanical degradation as defined by the decrease in kinematic viscosity for a specified reference oil after 30 shear cycles through the apparatus.

As specified in ASTM 6278, reference oil RL233 was purchased from the ASTM Test Monitoring Center and sheared through 30 cycles in the injector apparatus. 
The nozzle pressure was adjusted until the permanent viscosity loss at $100^{\circ} \mathrm{C}$ as measured by capillary viscometer was measured to be between $2.7-2.9$ cSt [50]. Several calibration tests were also completed (30 SC-E and 30 SC-F) in between shear tests of the blended lubricant to ensure that the shearing process remained consistent. Calibration test results are shown in Table 4.1.

Table 4.1: Summary of calibration tests with reference oil RL233 after 30 shear cycles (30 SC) through the injector apparatus. A nozzle pressure of 2200 psi produced a sufficient amount of degradation in the reference oil to proceed with shear stability testing of blended lubricants.

\begin{tabular}{r|cccc} 
Sample & NOP (psi) & Average efflux time $(\mathrm{s})$ & $\nu_{100^{\circ} \mathrm{C}}(\mathrm{cSt})$ & $\mathrm{PVL}(\mathrm{cSt})$ \\
\hline Unsheared & - & 420.7 & 14.67 & - \\
$30 \mathrm{SC}-\mathrm{A}$ & 1890 & 343.4 & 11.98 & 2.68 \\
$30 \mathrm{SC}-\mathrm{B}$ & 1990 & 343.5 & 11.98 & 2.68 \\
$30 \mathrm{SC}-\mathrm{C}$ & 2200 & 340.8 & 11.89 & 2.77 \\
$30 \mathrm{SC}-\mathrm{D}$ & 2200 & 341.4 & 11.91 & 2.75 \\
$30 \mathrm{SC}-\mathrm{E}$ & 2200 & 339.6 & 11.85 & 2.82 \\
$30 \mathrm{SC}-\mathrm{F}$ & 2200 & 341.2 & 11.90 & 2.77
\end{tabular}

\subsection{Shear Stability of Blended Lubricants}

Kinematic viscosity measurements of sheared samples indicated a small but measurable decrease in viscosity due to mechanical degradation from the injector apparatus. The decrease in kinematic viscosity after shearing was also proportionally greater for the higher molecular weight EC100 sample than the lower molecular weight EC45 sample as summarized in Table 4.2. In both cases the viscosity index was essentially unchanged - the ethyl cellulose polymer additive still functioned well as a VII after mechanical shearing even though there was a measurable decrease in the effect of polymer thickening for a blended lubricant. 
Although the chemical structure of the VII additive may influence certain solution properties of the blended lubricant, it has been widely confirmed that the shear stability of polymer additives is primarily determined by the length of the main polymer chain (which is characterized by the polymer's molecular weight) [2]. Both the magnitude of permanent viscosity loss and the shear stability index are comparable to typical shear stability results for polymer additives commonly used in petroleum-based lubricants as summarized in Table 4.2, where higher molecular weight polymers experienced a greater magnitude of permanent viscosity loss after mechanical shearing. While many traditional VIIs used in petroleum lubricants suffer from a need to balance the molecular weight and the polymer concentration (higher molecular weight polymers are able to effectively function as VIIs at low concentrations, but are more susceptible to permanent loss of viscosity after shearing, and vice versa), ethyl cellulose polymers may offer a high thickening efficiency at low concentrations with a molecular weight which is low enough to avoid the performance degradation from excessive decrease in polymer molecular weight due to mechanical shear. 
Table 4.2: Summary of kinematic viscosity changes for blended lubricants after 30 shear cycles (30 SC). Comparison results are also shown for a study which investigated the shear degradation of four separate molecular weights of PMMA (identified as MA1 to MD1 in the study) additive in mineral oil [4].

\begin{tabular}{|c|c|c|c|c|c|c|c|}
\hline Sample & $M_{W}$ & c (wt.\%) & $\nu_{40^{\circ} \mathrm{C}}(\mathrm{cSt})$ & $\nu_{100^{\circ} \mathrm{C}}(\mathrm{cSt})$ & VI & PVL & SSI \\
\hline EC45 & $68960[3]$ & \multirow{2}{*}{1.0} & 184.2 & 21.87 & 142 & - & - \\
\hline EC45 $30 \mathrm{SC}$ & - & & 183.2 & 21.71 & 142 & 0.73 & 1.56 \\
\hline EC100 & $76530[3]$ & \multirow{2}{*}{1.0} & 217.6 & 25.78 & 151 & - & - \\
\hline EC100 $30 \mathrm{SC}$ & - & & 206.9 & 24.86 & 150 & 3.57 & 6.51 \\
\hline MA1 & 490000 & \multirow{2}{*}{3.40} & 77.84 & 14.53 & 196 & - & - \\
\hline MA1 $30 \mathrm{SC}$ & - & & - & 12.01 & 196 & 16.99 & 30.66 \\
\hline MB1 & 133000 & \multirow{2}{*}{7.13} & 78.27 & 14.50 & 194 & - & - \\
\hline MB1 $30 \mathrm{SC}$ & - & & - & 13.56 & 193 & 6.35 & 11.47 \\
\hline MC1 & 70000 & \multirow{2}{*}{10.15} & 86.43 & 14.57 & 176 & - & - \\
\hline MC1 $30 \mathrm{SC}$ & - & & - & 14.09 & 176 & 3.16 & 5.81 \\
\hline MD1 & 45000 & \multirow{2}{*}{15.54} & 94.32 & 14.51 & 160 & - & - \\
\hline MD1 $30 \mathrm{SC}$ & - & & - & 14.25 & 160 & 1.72 & 3.17 \\
\hline
\end{tabular}

In order to determine the changes of intrinsic viscosity after mechanical shearing, it was necessary to prepare diluted samples for both the sheared and unsheared oil. As described in Chapter 3, the samples of mechanically sheared $1 \%$ wt. concentration were diluted in base oil to concentrations of $0.75 \%$ wt, $0.50 \%$ wt, and $0.25 \%$ wt. Kinematic viscosity and density measurements at a given polymer concentration and temperature allowed for the determination of absolute viscosity for use in subsequent calculations of solution properties. The measured kinematic and dynamic viscosities of the diluted samples are shown in Table 4.3. 
Table 4.3: Kinematic and dynamic viscosities of diluted lubricant samples with ethyl cellulose (EC) viscosity index additives before and after 30 cycles (30 SC) of mechanical degradation

\begin{tabular}{rr|rrrr} 
Sample & $\mathrm{c}($ wt. \%) & $\nu_{40}{ }^{\circ} \mathrm{C}$ & $\nu_{100^{\circ} \mathrm{C}}$ & $\eta_{40{ }^{\circ} \mathrm{C}}$ & $\eta_{100^{\circ} \mathrm{C}}$ \\
\hline & $1.0 \%$ & 184.2 & 21.87 & 0.1696 & 0.0193 \\
EC45 & $0.75 \%$ & 153.6 & 18.79 & 0.1414 & 0.0166 \\
& $0.50 \%$ & 126.4 & 16.08 & 0.1162 & 0.0142 \\
& $0.25 \%$ & 103.5 & 13.71 & 0.0952 & 0.0121 \\
\hline \multirow{2}{*}{ EC45 30 SC } & $1.0 \%$ & 183.2 & 21.71 & 0.1687 & 0.0191 \\
& $0.75 \%$ & 152.8 & 18.70 & 0.1406 & 0.0165 \\
& $0.50 \%$ & 126.0 & 15.98 & 0.1159 & 0.0141 \\
& $0.25 \%$ & 103.4 & 13.68 & 0.0950 & 0.0120 \\
\hline \multirow{2}{*}{ EC100 } & $1.0 \%$ & 217.6 & 25.78 & 0.2004 & 0.0227 \\
& $0.75 \%$ & 173.9 & 21.20 & 0.1600 & 0.0187 \\
& $0.50 \%$ & 138.7 & 17.47 & 0.1276 & 0.0154 \\
& $0.25 \%$ & 108.6 & 14.28 & 0.0998 & 0.0126 \\
\hline \multirow{2}{*}{ EC100 30 SC } & $1.0 \%$ & 206.9 & 24.86 & 0.1905 & 0.0219 \\
& $0.75 \%$ & 167.8 & 20.76 & 0.1544 & 0.0183 \\
& $0.50 \%$ & 134.9 & 17.20 & 0.1240 & 0.0152 \\
& $0.25 \%$ & 107.2 & 14.17 & 0.0986 & 0.0125 \\
& & & & &
\end{tabular}

The effect of polymer concentration on the magnitude of thickening is described by its relative viscosity as defined in Table 2.5. The specific viscosity is also a useful indicator of the increase in solution viscosity which can be attributed to the addition of polymer additives. Tabulated results for the relative and specific viscosities of sheared and unsheared diluted samples are shown in Table 4.4. After mechanical shearing, there was a measurable decrease in the relative viscosity across all temperatures and solution concentrations as shown in Figure 4.1 and Figure 4.2. The magnitude of relative viscosity loss was greater for the EC100 sample than the EC45 sample at $40^{\circ} \mathrm{C}$ and $100^{\circ} \mathrm{C}$ which again indicates a greater susceptibility to 
shear degradation for the higher molecular weight polymer.

Table 4.4: Relative and specific viscosities of diluted lubricant samples with ethyl cellulose (EC) viscosity index additives before and after 30 cycles (30 SC) of mechanical degradation

\begin{tabular}{rr|rccc} 
Sample c (wt. \%) & $\eta_{\text {rel }, 40{ }^{\circ} \mathrm{C}}$ & $\eta_{\text {rel }, 100^{\circ} \mathrm{C}}$ & $\eta_{s p, 40{ }^{\circ} \mathrm{C}}$ & $\eta_{s p, 100^{\circ} \mathrm{C}}$ \\
\hline & $1.0 \%$ & 2.195 & 1.880 & 1.195 & 0.880 \\
EC45 & $0.75 \%$ & 1.830 & 1.615 & 0.830 & 0.615 \\
& $0.50 \%$ & 1.505 & 1.381 & 0.505 & 0.381 \\
& $0.25 \%$ & 1.232 & 1.177 & 0.232 & 0.177 \\
\hline \multirow{2}{*}{ EC45 30 SC } & $1.0 \%$ & 2.184 & 1.866 & 1.184 & 0.866 \\
& $0.75 \%$ & 1.820 & 1.607 & 0.820 & 0.607 \\
& $0.50 \%$ & 1.500 & 1.373 & 0.500 & 0.373 \\
& $0.25 \%$ & 1.230 & 1.175 & 0.230 & 0.175 \\
\hline \multirow{2}{*}{ EC100 } & $1.0 \%$ & 2.594 & 2.216 & 1.594 & 1.216 \\
& $0.75 \%$ & 2.071 & 1.822 & 1.071 & 0.822 \\
& $0.50 \%$ & 1.651 & 1.501 & 0.651 & 0.501 \\
& $0.25 \%$ & 1.292 & 1.226 & 0.292 & 0.226 \\
\hline & $1.0 \%$ & 2.466 & 2.137 & 1.466 & 1.137 \\
& $0.75 \%$ & 1.999 & 1.784 & 0.999 & 0.784 \\
& $0.50 \%$ & 1.606 & 1.478 & 0.606 & 0.478 \\
& $0.25 \%$ & 1.276 & 1.217 & 0.276 & 0.217 \\
& & & & &
\end{tabular}




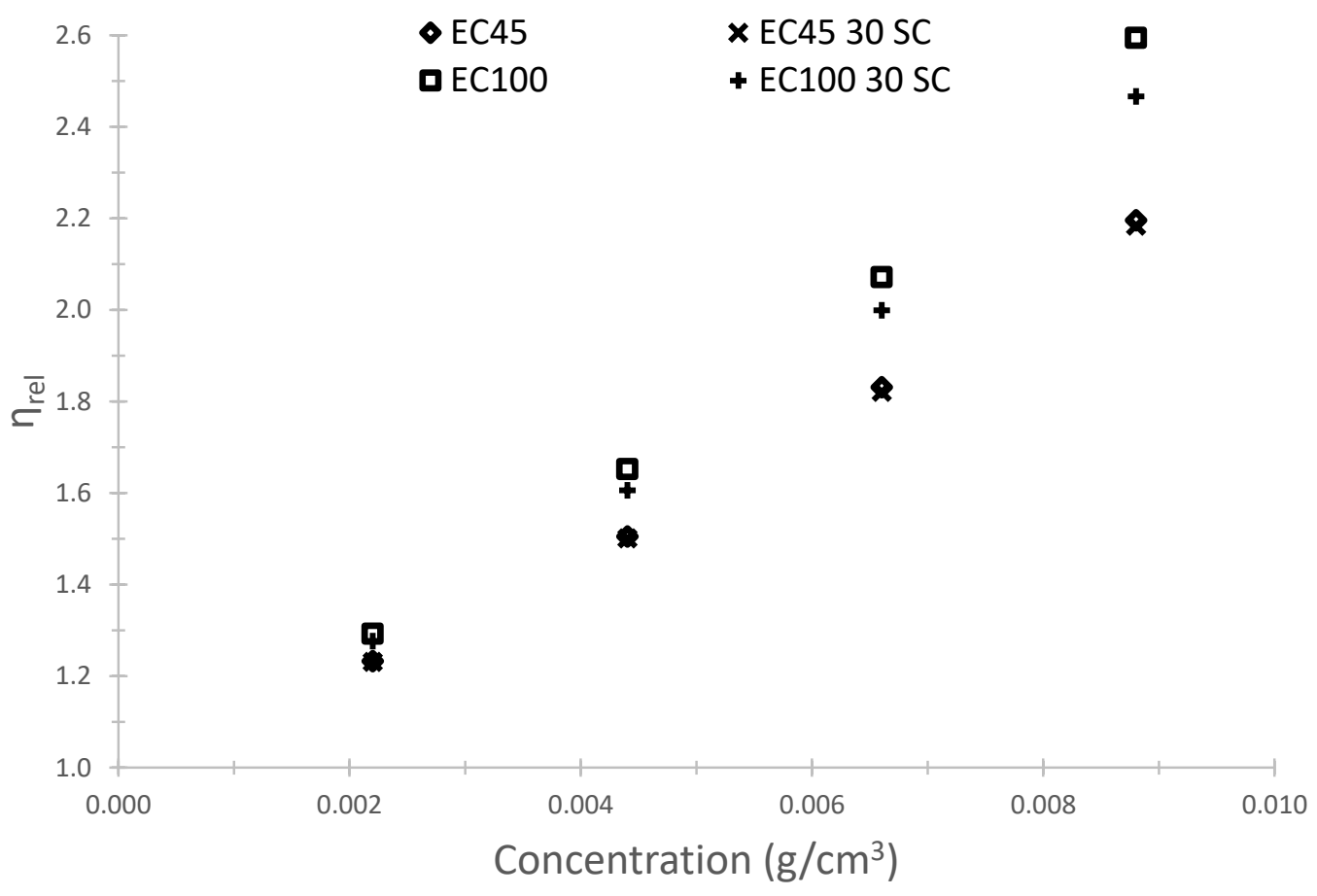

Figure 4.1: Relative viscosity of diluted sheared and unsheared samples at $40^{\circ} \mathrm{C}$

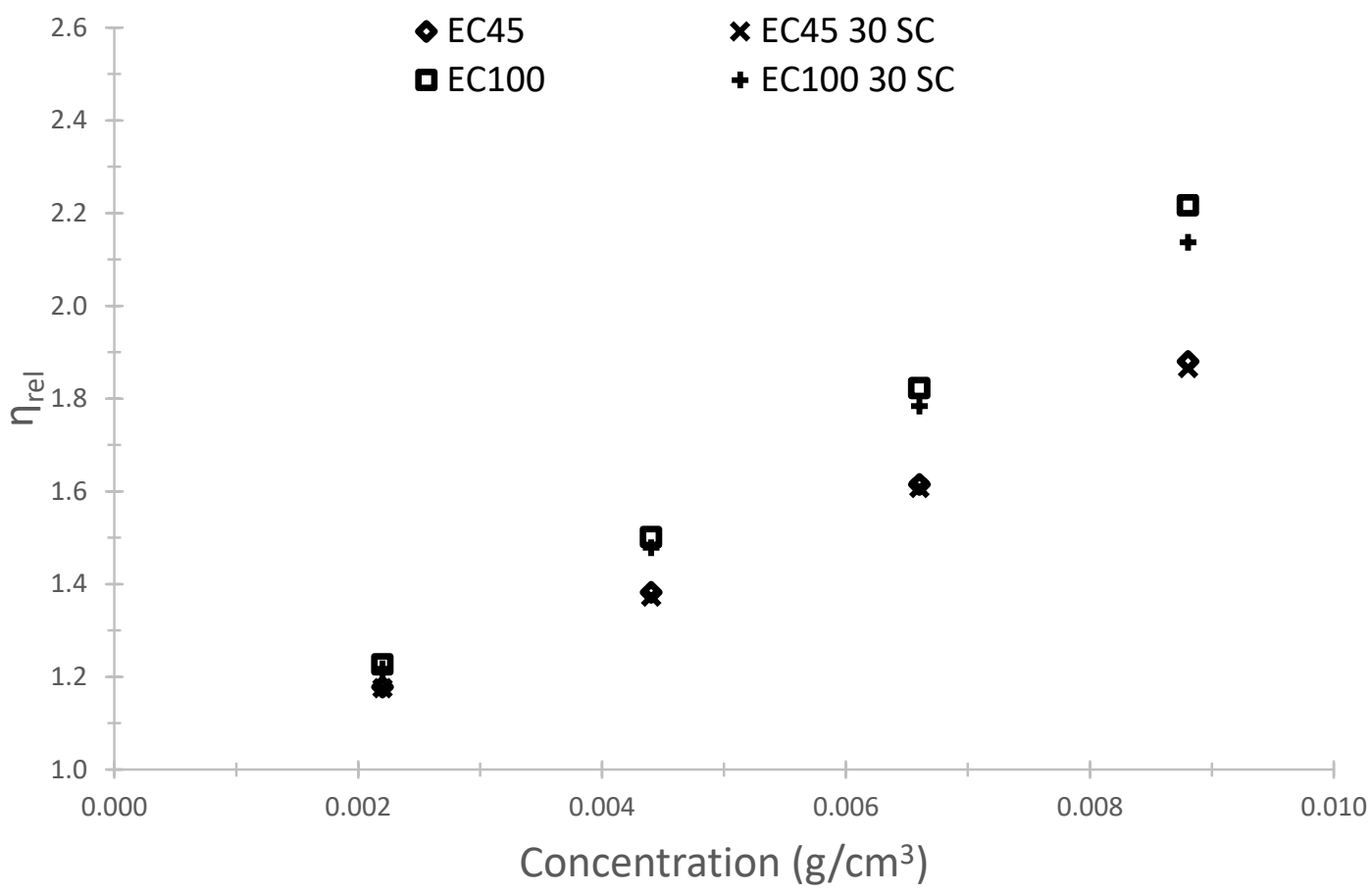

Figure 4.2: Relative viscosity of diluted sheared and unsheared samples at $100^{\circ} \mathrm{C}$ 
The intrinsic viscosity for each sample was estimated as described in Section 3.4.2. Results are tabulated in Tables 4.5 and 4.6, and individual plots are included in Appendix A. With this method, changes in the intrinsic viscosity due to mechanical shearing appeared to follow a consistent trend where the higher molecular weight polymer blend of EC100 experienced a proportionally greater decrease in the intrinsic viscosity as summarized in Table 4.9. It is apparent from the data that the shear degradation process resulted in a decrease in the effectiveness for the blended polymer to increase the viscosity of the formulated lubricant. Although the molecular weight of the degraded samples was never determined, the observed decrease in the intrinsic viscosity likely corresponds to a decrease in the polymer molecular weight due to mechanical shearing. 
Table 4.5: Intrinsic viscosity as determined by a linear regression of reduced viscosity measurements on diluted lubricant samples with ethyl cellulose (EC) viscosity index additives before and after 30 cycles (30 SC) of mechanical degradation

\begin{tabular}{|c|c|c|c|c|c|}
\hline Sample & c (wt. \%) & $\eta_{\text {red }, 40^{\circ} \mathrm{C}}$ & $\eta_{\text {red }, 100^{\circ} \mathrm{C}}$ & {$[\eta]_{H, 40^{\circ} \mathrm{C}}$} & {$[\eta]_{H, 100^{\circ} \mathrm{C}}$} \\
\hline \multirow{4}{*}{ EC45 } & $1 \%$ & 130.1 & 99.91 & \multirow{4}{*}{90.84} & \multirow{4}{*}{73.99} \\
\hline & $0.75 \%$ & 120.5 & 93.07 & & \\
\hline & $0.50 \%$ & 109.9 & 86.63 & & \\
\hline & $0.25 \%$ & 100.9 & 80.63 & & \\
\hline \multirow{4}{*}{ EC45 $30 \mathrm{SC}$} & $1 \%$ & 128.9 & 98.35 & \multirow{4}{*}{90.15} & \multirow{4}{*}{72.63} \\
\hline & $0.75 \%$ & 119.1 & 91.89 & & \\
\hline & $0.50 \%$ & 108.8 & 84.68 & & \\
\hline & $0.25 \%$ & 100.2 & 79.46 & & \\
\hline \multirow{4}{*}{ EC100 } & $1 \%$ & 173.5 & 138.1 & \multirow{4}{*}{111.26} & \multirow{4}{*}{90.6} \\
\hline & $0.75 \%$ & 155.5 & 124.4 & & \\
\hline & $0.50 \%$ & 141.8 & 113.7 & & \\
\hline & $0.25 \%$ & 127.1 & 102.8 & & \\
\hline \multirow{4}{*}{ EC100 30 SC } & $1 \%$ & 159.6 & 129.1 & \multirow{4}{*}{106.26} & \multirow{4}{*}{88.3} \\
\hline & $0.75 \%$ & 144.9 & 118.7 & & \\
\hline & $0.50 \%$ & 131.9 & 108.6 & & \\
\hline & $0.25 \%$ & 120.1 & 98.58 & & \\
\hline
\end{tabular}


Table 4.6: Intrinsic viscosity as determined by a linear regression of inherent viscosity measurements on diluted lubricant samples with ethyl cellulose (EC) viscosity index additives before and after 30 cycles (30 SC) of mechanical degradation

\begin{tabular}{|c|c|c|c|c|c|}
\hline Sample & c (wt. \%) & $\eta_{i n h, 40^{\circ} \mathrm{C}}$ & $\eta_{i n h, 100^{\circ} \mathrm{C}}$ & {$[\eta]_{K, 40^{\circ} \mathrm{C}}$} & {$[\eta]_{K, 100^{\circ} \mathrm{C}}$} \\
\hline \multirow{4}{*}{ EC45 } & $1 \%$ & 85.59 & 71.68 & \multirow{4}{*}{92.47} & \multirow{4}{*}{75.08} \\
\hline & $0.75 \%$ & 87.72 & 72.55 & & \\
\hline & $0.50 \%$ & 88.97 & 73.39 & & \\
\hline & $0.25 \%$ & 90.78 & 74.22 & & \\
\hline \multirow{4}{*}{ EC45 $30 \mathrm{SC}$} & $1 \%$ & 85.01 & 70.84 & \multirow{4}{*}{91.77} & \multirow{4}{*}{73.79} \\
\hline & $0.75 \%$ & 86.94 & 71.82 & & \\
\hline & $0.50 \%$ & 88.24 & 71.97 & & \\
\hline & $0.25 \%$ & 90.15 & 73.23 & & \\
\hline \multirow{4}{*}{ EC100 } & $1 \%$ & 103.7 & 90.36 & \multirow{4}{*}{114.24} & \multirow{4}{*}{93.61} \\
\hline & $0.75 \%$ & 105.7 & 90.82 & & \\
\hline & $0.50 \%$ & 109.2 & 92.19 & & \\
\hline & $0.25 \%$ & 111.5 & 92.69 & & \\
\hline \multirow{4}{*}{ EC100 $30 \mathrm{SC}$} & $1 \%$ & 98.24 & 86.23 & \multirow{4}{*}{108.5} & \multirow{4}{*}{90.47} \\
\hline & $0.75 \%$ & 100.5 & 87.65 & & \\
\hline & $0.50 \%$ & 103.1 & 88.74 & & \\
\hline & $0.25 \%$ & 106.1 & 89.22 & & \\
\hline
\end{tabular}

With curve fitting tools available in MATLAB, a least-squares second order polynomial was also fitted to the diluted solution properties described in Table 4.4. In order to improve the quality of the curve fit, a boundary condition was established such that the specific viscosity was equal to zero when the polymer concentration was zero. The Huggins and Kraemer coefficients (as defined in Equations 2.4 and 2.5), as well as the intrinsic viscosity, were estimated and summarized in Table 4.7 and Table 4.8. The change of intrinsic viscosity obtained from the second order polynomial curve fit generally observed the same trend as the change of intrinsic viscosity as obtained from methods described by ASTM 2857. The higher 
molecular weight EC100 blend was observed to experience a greater degradation in the effectiveness for the polymer additive to increase the solution viscosity than the lower molecular weight EC45 blend.

Table 4.7: Huggins coefficients and intrinsic viscosity of diluted lubricant samples with ethyl cellulose (EC) viscosity index additives before and after 30 cycles (30 SC) of mechanical degradation. Coefficients were estimated from a second order polynomial curve fit of the specific viscosity based on Equation 2.4

\begin{tabular}{r|cccccc} 
Sample & $K_{H, 40^{\circ} \mathrm{C}}$ & $K_{H, 100^{\circ} \mathrm{C}}$ & {$[\eta]_{H, 40^{\circ} \mathrm{C}}$} & {$[\eta]_{H, 100^{\circ} \mathrm{C}}$} & $\mathrm{TSS}_{40^{\circ} \mathrm{C}}$ & $\mathrm{TSS}_{100^{\circ} \mathrm{C}}$ \\
\hline EC45 & 0.5253 & 0.5681 & 94.52 & 73.15 & 0.90 & 0.48 \\
EC45 30 SC & 0.5408 & 0.5829 & 93.16 & 71.87 & 0.88 & 0.47 \\
EC100 & 0.6110 & 0.7276 & 112.4 & 88.02 & 1.59 & 0.93 \\
EC100 30 SC & 0.5595 & 0.6046 & 108.3 & 87.98 & 1.35 & 0.81
\end{tabular}

Table 4.8: Kraemer coefficients and intrinsic viscosity of diluted lubricant samples with ethyl cellulose (EC) viscosity index additives before and after 30 cycles (30 SC) of mechanical degradation. Coefficients were estimated from a second order polynomial curve fit of the relative viscosity based on the Equation 2.5

\begin{tabular}{r|rccccc} 
Sample & $K_{K, 40^{\circ} C}$ & $K_{K, 100^{\circ} C}$ & {$[\eta]_{K, 40^{\circ} C}$} & {$[\eta]_{K, 100^{\circ} C}$} & $\operatorname{TSS}_{40^{\circ} C}$ & $\operatorname{TSS}_{100^{\circ} C}$ \\
\hline EC45 & -0.0912 & -0.0665 & 97.01 & 75.06 & 0.90 & 0.48 \\
EC45 30 SC & -0.0874 & -0.0597 & 95.89 & 73.80 & 0.88 & 0.47 \\
EC100 & -0.0846 & -0.0414 & 118.6 & 93.53 & 1.59 & 0.93 \\
EC100 30 SC & -0.0915 & -0.0669 & 112.7 & 91.33 & 1.35 & 0.81
\end{tabular}

Table 4.9: Percent decrease of the intrinsic viscosity of blended lubricants after shearing as determined by a second order polynomial curve fit of the Huggins and Kraemer equations, and a linear approximation (ASTM 2857).

\begin{tabular}{r|cccc|cccc} 
& \multicolumn{3}{|c|}{ Second order polynomial curve fit } & \multicolumn{4}{|c}{ ASTM 2857} \\
Sample & {$[\eta]_{H, 40^{\circ} \mathrm{C}}$} & {$[\eta]_{K, 40^{\circ} \mathrm{C}}$} & {$[\eta]_{H, 100^{\circ} \mathrm{C}}$} & {$[\eta]_{K, 100^{\circ} \mathrm{C}}$} & {$[\eta]_{H, 40^{\circ} \mathrm{C}}$} & {$[\eta]_{K, 40^{\circ} \mathrm{C}}$} & {$[\eta]_{H, 100^{\circ} \mathrm{C}}$} & {$[\eta]_{K, 100^{\circ} \mathrm{C}}$} \\
\hline EC45 & 1.43 & 1.15 & 1.75 & 1.68 & 0.76 & 0.76 & 1.84 & 1.72 \\
EC100 & 3.65 & 4.97 & 0.05 & 2.35 & 4.49 & 5.02 & 2.56 & 3.35
\end{tabular}




\section{Chapter 5}

\section{Conclusions and Recommendations for Future Work}

\subsection{Summary of Experimental Results}

A diesel fuel injector apparatus was successfully re-purposed and modified to enable a continuous circulation of lubricating oil through the fuel injector nozzle for the purpose of examining the shear stability of polymer VIIs as described by ASTM 6278. Modifications from previous efforts included the installation of parallel air-pressure amplifiers and staged accumulators to ensure a consistent supply of pressurized air into the pneumatic actuator.

The shear stability of vegetable oil based lubricants blended with two molecular weight ethyl cellulose polymers was examined in detail by measuring the kinematic viscosity at $40^{\circ} \mathrm{C}$ and $100^{\circ} \mathrm{C}$ of samples before and after mechanical degradation. The magnitude of shear stability was determined with a comparison of the percentage of permanent viscosity loss and the shear stability index, as well as changes in the solution properties of blended lubricants. 
Ethyl cellulose polymers were demonstrated to offer a high degree of thickening at relatively low polymer concentrations and low molecular weights when compared to traditional VIIs used in petroleum-based lubricants. With a relatively stable shear behaviour and desirable solution properties, low molecular weight ethyl cellulose polmyers may be suitable as a viscosity index additive when blended with vegetable oil-based lubricants.

\subsection{Recommendations for Future Work}

Determination of the lubricant's percentage of permanent viscosity loss and the shear stability index was useful for characterizing the changes in the lubricant viscosity after mechanical degradation. However, these metrics do not provide any direct indication as to how the polymer molecules are physically affected by the process. Although measurements of the change of intrinsic viscosity provided some insight and suggest an overall decrease in polymer molecular weight, it was not possible to draw specific conclusions regarding the change in the polymer molecular weight which is the primary physical quantity that determines the effectiveness of a polymer to function as a VII. It is recommended that any future study on this matter include a determination of the changes in polymer molecular weight distribution and the polydispersity index of the polymer additive.

As previously stated in Section 2.5, the diesel fuel injector experiment is just one of a variety of methods which may be used to examine the shear stability of polymer additives in blended lubricants. As there is some debate regarding the limiting shear viscosity, future study may also examine any bias in the diesel fuel injector apparatus by subjecting the polymer containing lubricants to the sonic shear stability test or the high-temperature-high-shear test which are also described by ASTM 2603 and 
ASTM 5481.

Additionally, it should be stressed that the ethyl cellulose polymer used in this study was selected based on experiments conducted by other researchers. From this study it may be concluded that ethyl cellulose functioned well as a VII in terms of its shear stability, but there may be many other polymers (either cellulose-based, or based on a different family of biodegradable polymers) which may also function well as a VII. Further study could focus efforts on categorizing the shear stability performance of several different families of polymer additives. 


\section{References}

[1] S Boyde. Green lubricants. environmental benefits and impacts of lubrication. Green Lubrication, 2002.

[2] LR Rudnick, editor. Lubricant Additives : Chemistry and Applications. CRC Press, 2009.

[3] M.A Delgado, L.A Quinchia, H.A Spikes, and C Gallegos. Suitability of ethyl cellulose as multifunctional additive for blends of vegetable oil-based lubricants. Journal of Cleaner Production, 2017.

[4] W Bartz. Influence of viscosity index improver, molecular weight, and base oil on thickening, shear stability, and evaporation losses of multigrade oils. Lubrication Science, 2000.

[5] PL Menezes, M Nosonovsky, SP Ingole, SV Kailas, and MR Lovell. Tribology for Scientists and Engineers. Springer, 2013.

[6] Sigma aldrich product description.

[7] H.G Muller. Mechanism of action of viscosity index improvers. Tribology International, 1978.

[8] R.M Mortier, M.F Fox, and S.T Orszulik, editors. Chemistry and Technology of Lubricants. 3rd ed. Springer, 2010.

[9] JL Herbeaux, A Flamberg, RD Koller, and WE VanArsdale. Assesment of shear degradation simulators. SAE Technical Papers, 1998.

[10] S.G. Daviault. Characterization of the fuel injection process within the ignition quality tester (iqt ${ }^{T M}$. Master's thesis, Carleton University, 2011.

[11] ASTM D445-15 : Standard test method for kinematic viscosity of transparent and opaque liquids. 2015. 
[12] ASTM D2857-16 : Standard practice for dilute solution viscosity of polymers. 2016.

[13] G.E Totten, editor. ASTM Fuels and Lubricants Handbook. ASTM International, 2003.

[14] L Wood. The change of viscosity of oils containing high polymers when subjected to high rates of shear. British Journal of Applied Physics, 1950.

[15] D Dowson. History of Tribology. Longman, 1979.

[16] W Bartz. Lubricants and the environment. Tribology International, 1998.

[17] 1999 Levizzari. Environmental and economic impact of re-refined products : a lifecycle analysis. In Proc. of the 6th International LFE Conference, Brussels, 1999.

[18] M et al Stalmans. European lifecycle inventory for detergent surfactants production. Tenside Surfactants Detergents, 1995.

[19] National Research Council. Oil in the sea : Inputs, fates, and effects. National Academy Press, 2003.

[20] YE Yong. Effects of used lubricating oil on two mangroves aegiceras corniculatum and avicennia marina. Journal of Environmental Sciences, 2007.

[21] D Horner. Recent trends in environmentally friendly lubricants. Journal of Synthetic Lubricants, 2002.

[22] J Hailing. Environmentally friendly lubrication issues. Wear, 1991.

[23] Coordinating European Council for the Development of Performance Tests for Lubricants and Engine Fuels. Test method for the biodegradability of two-stroke cycle outboard engine oils in water. CLC-L-33-T-93, 1993.

[24] Organization for Economic Cooperation and Development. Oecd guideline for the testing of chemicals. ready biodegradability. OECD301, 1992.

[25] P Anastas and R Lankey. Life cycle assessment and green chemistry : the yin and yang of industrial ecology. Green Chemistry, 2000.

[26] P De Caro and A Gaset. Lubricants based on renewable resources. Oilseeds and fats, Crops and Lipids, 1999. 
[27] S Erhan and S Asadauskas. Lubricant basestocks from vegetable oils. Industrial Crops and Products, 2000.

[28] RK Hewstone. Environmental health aspects of lubricant additives. The Science of the Total Environment, 1994.

[29] International Organization for Standardization (ISO). Industrial oils and related products (class 1) - classification - part 4: Family h (hydraulic systems). ISO67434-2015, 2015.

[30] T Panchal, A Patel, and D Chauhan. A methodological review on bio-lubricants fro vegetable oil based resources. Renewable and Sustainable Energy Reviews, 2017.

[31] LR Rudnick, editor. Synthetics, Mineral Oils, and Bio-Based Lubricants : Chemistry and Technology. 2nd ed. CRC Press, 2013.

[32] L.A. Quinchia, M.A. Delgado, J.M Franco, H.A Spikes, and C Gallegos. Lowtemperature flow behaviour of vegetable oil-based lubricants. Industrial Crops and Products, 2012.

[33] P Miles. Synthetics versus vegetable oils: Applications, options, and performance. Journal of Synthetic Lubrication, 1998.

[34] L.A. Quinchia, M.A. Delgado, T Reddyhoff, C Gallegos, and H.A Spikes. Tribological studies of potential vegetable oil-based lubricants containing environmentally friendly viscosity modifiers. Tribology International, 2014.

[35] A Biresaw, S Adhvaryu, and S Erhan. Friction properties of vegetable oils. Journal of the American Oil Chemists Society, 2003.

[36] S Soni and M Agarwal. Lubricants from renewable resources - a review. Green Chemistry Letters and Reviews, 2014.

[37] L.A. Quinchia, M.A. Delgado, C Valencia, J.M Franco, and C Gallegos. Viscosity modification of high-oleic sunflower oil with polymeric additives for the design of new biolubricant formulations. Environmental Science and Technology, 2009.

[38] T Mang and W Dresel. Lubricants and Lubrication. Wiley, 2006.

[39] S Bair. The shear rheology of thin compressed liquid films. Proceedings of the Institution of Mechanical Engineers, Part 1 : Journal of Engineering Tribology, 2002. 
[40] ASTM D2270-10 : Standard practice for calculating viscosity index from kinematic viscosity at 40 and 1002010.

[41] M.M Khonsari and E.R Booser. Applied tribology : Bearing design and lubrication. Wiley, 2008.

[42] T.W. Selby. The non-newtonian characteristics of lubricating oils. ASLE Transactions, 1958.

[43] W.R. Moore. Entropy of activation of viscous flow in dilute solutions of high polymers. International Journal of Science, 1965.

[44] Commission directive 93/21/eec of 27 april 1993 adapting to technical progress for the 18th time council directive $67 / 548 /$ eec on the approximation of the laws, regulations, and administrative provisions relating to the classification, packaging, and labelling of dangerous substances. Official Journal of the European Commission, 1993.

[45] Product safety assessment : Ethocel ethyl cellulose polymers. DOW Chemicals, 2014.

[46] R Pamies and J.G.H Cifre. Determination of intrinsic viscosities of macromolecules and nanoparticles. comparison of single-point and dilution procedures. Colloid Polymer Science, 2008.

[47] K Mackenzie and A Jemmett. Polymer shear stability. Wear, 1971.

[48] C Bezot, C Hesse-Bezot, and D Faure. The effect of mechanical stress on four viscosity improver polymers. Lubrication Science, 2000.

[49] D Alexander, M Kapuscinski, and M Laffin. Shear stability index of vi improvers; its dependency on method of determination. Lubrication Engineering, 1989.

[50] ASTM D6278-12: Standard test method for shear stability of polymer containing fluids using a european diesel injector apparatus. 2012.

[51] ASTM D2603-01 : Standard test method for sonic shear stability of polymer containing oils. 2013.

[52] M Mortier. Laboratory shearing tests for viscosity index improvers. Tribotest Journal, 1996. 
[53] X Du, H Chen, and L Shui. Correlation study of different shear stability test methods for hydraulic oils. Society of Tribologists and Lubrication Engineers, 2015 .

[54] P Ghosh and G Karmakar. Evaluation of sunflower oil as a multifunctional lubricating oil additive. International Journal of Industrial Chemistry, 2014.

[55] F LaForge. The development of a diesel injector experiment to study the combined effects of oxidation and shear on lubricating oil viscosity. Master's thesis, Carleton University, 2015.

[56] Instructions for the use of the cannon-ubbelohde viscometer. 2002. 


\section{Appendix A}

\section{Dilution Plots for Determination of}

\section{Intrinsic Viscosity}

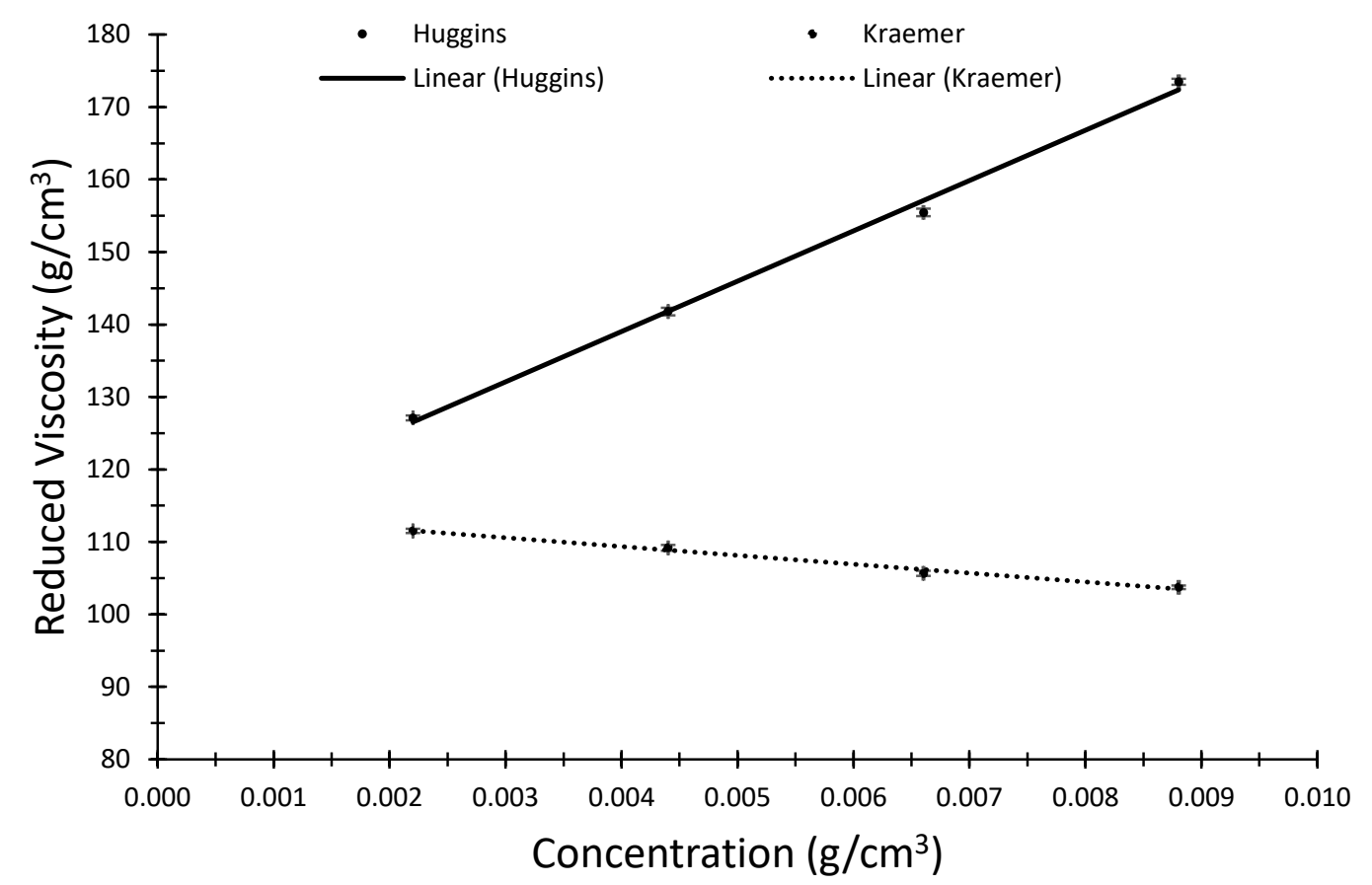

Figure A.1: EC100 Dilution at $40^{\circ} \mathrm{C}$ 


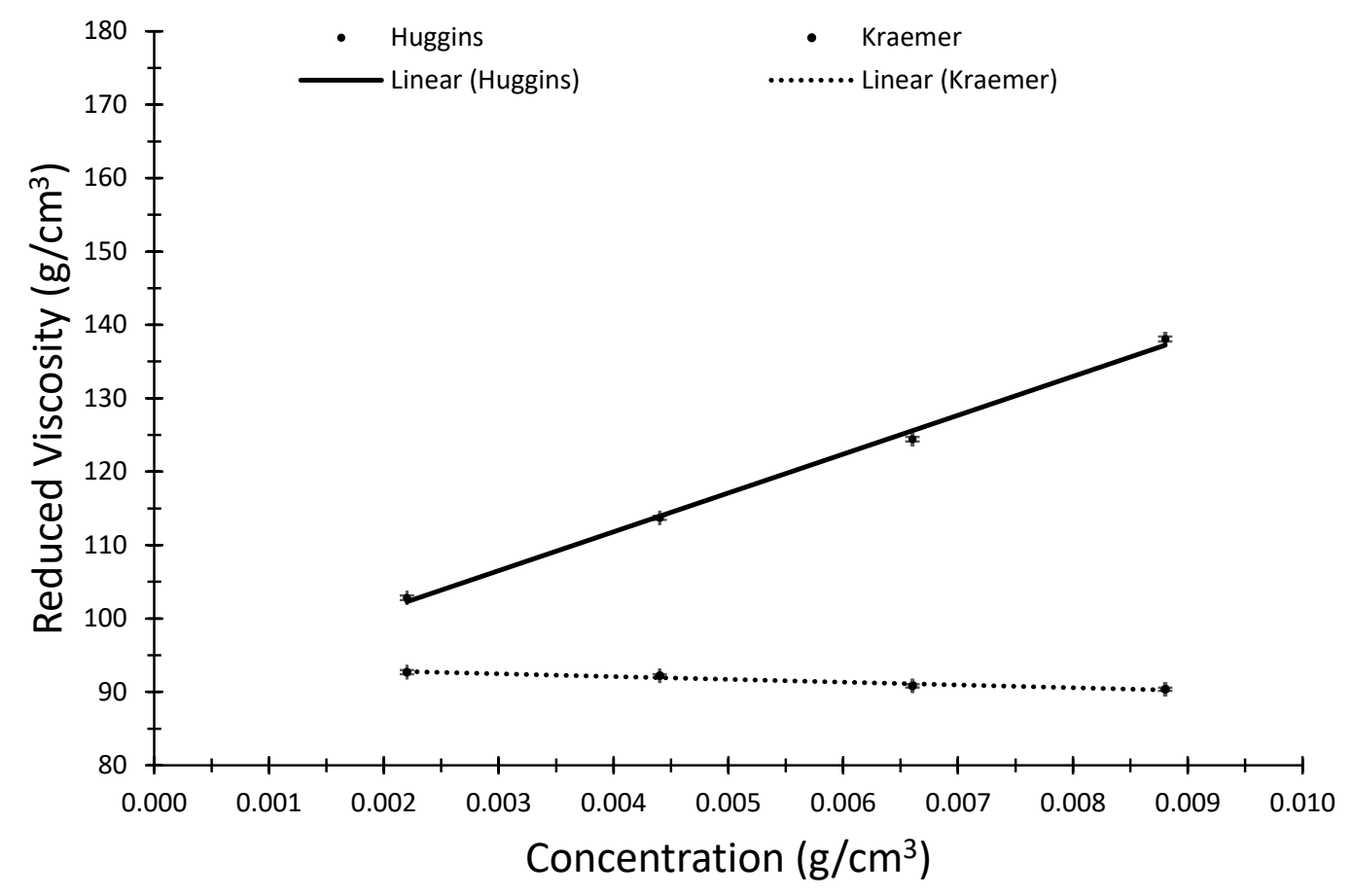

Figure A.2: EC100 Dilution at $100^{\circ} \mathrm{C}$

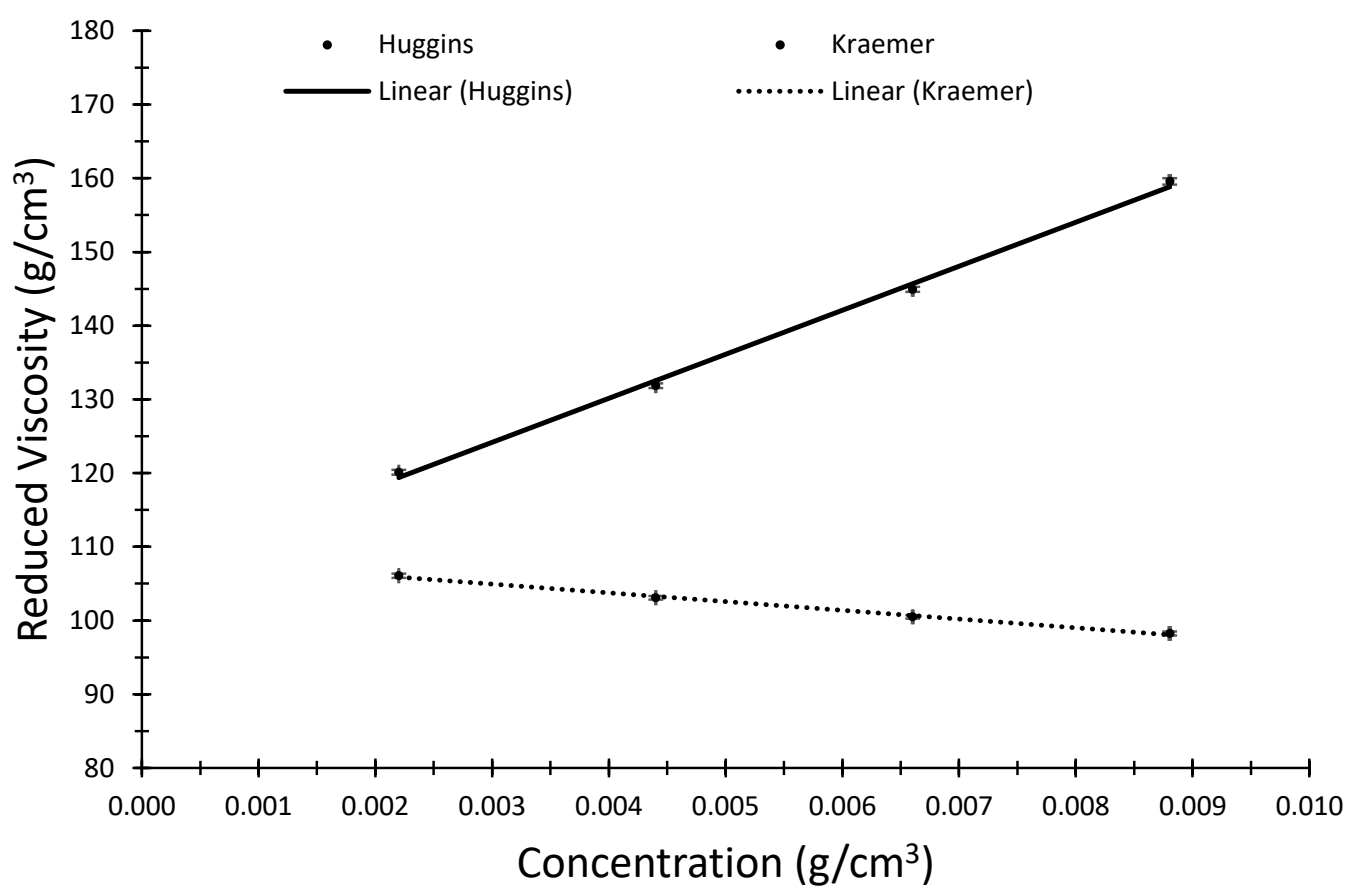

Figure A.3: EC100 30SC Dilution at $40^{\circ} \mathrm{C}$ 


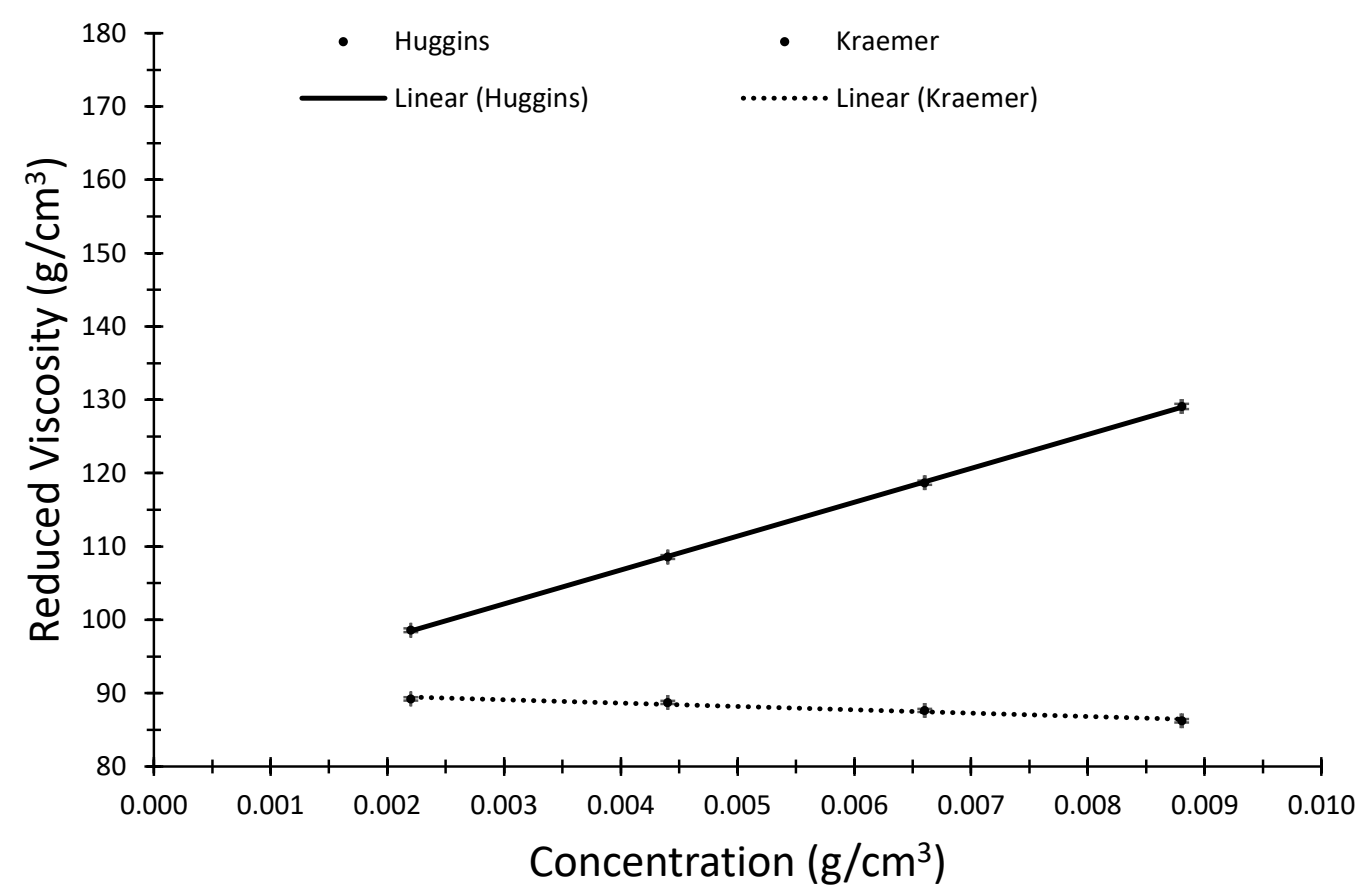

Figure A.4: EC100 30SC Dilution at $100^{\circ} \mathrm{C}$

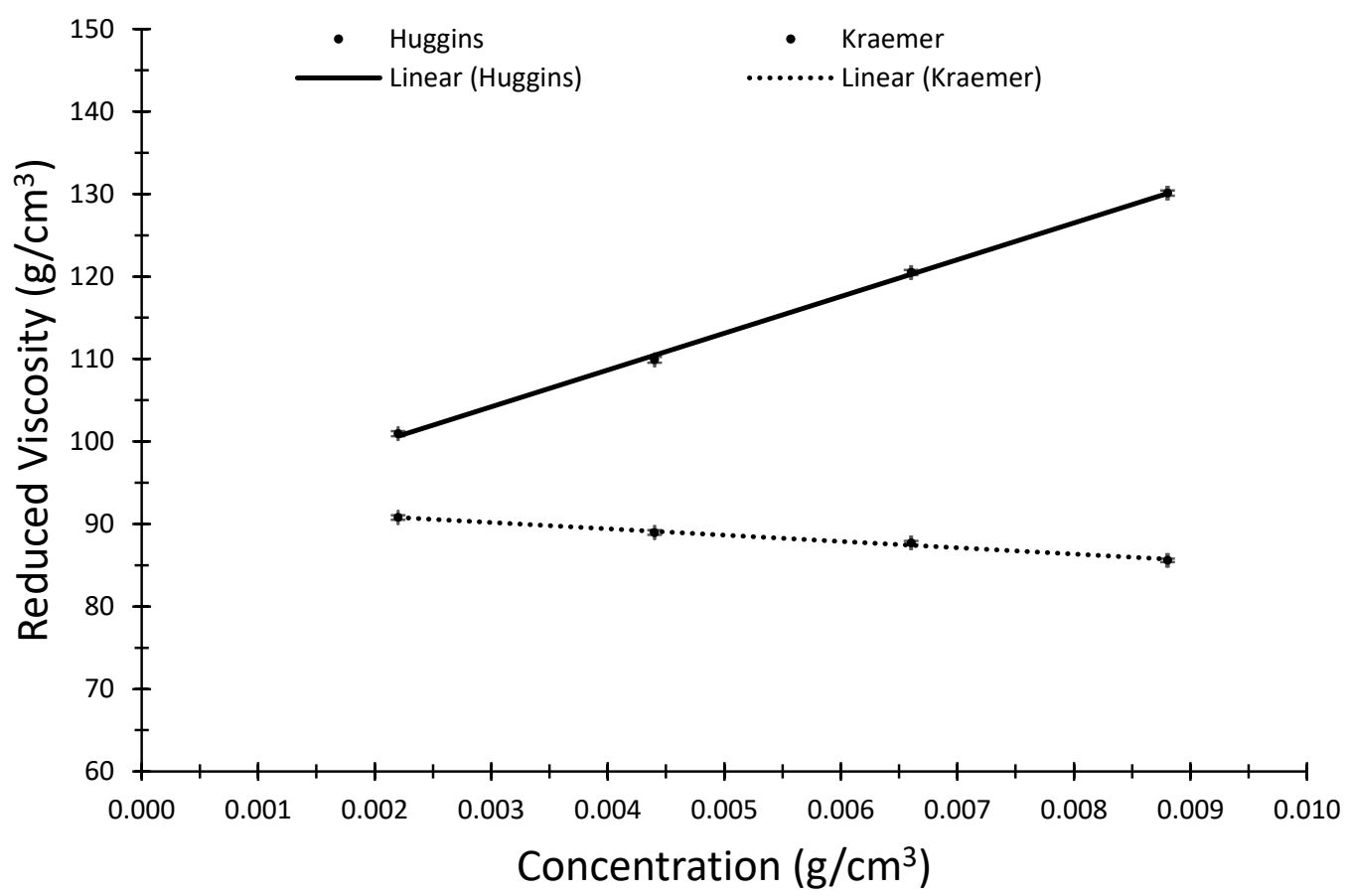

Figure A.5: EC45 Dilution at $40^{\circ} \mathrm{C}$ 


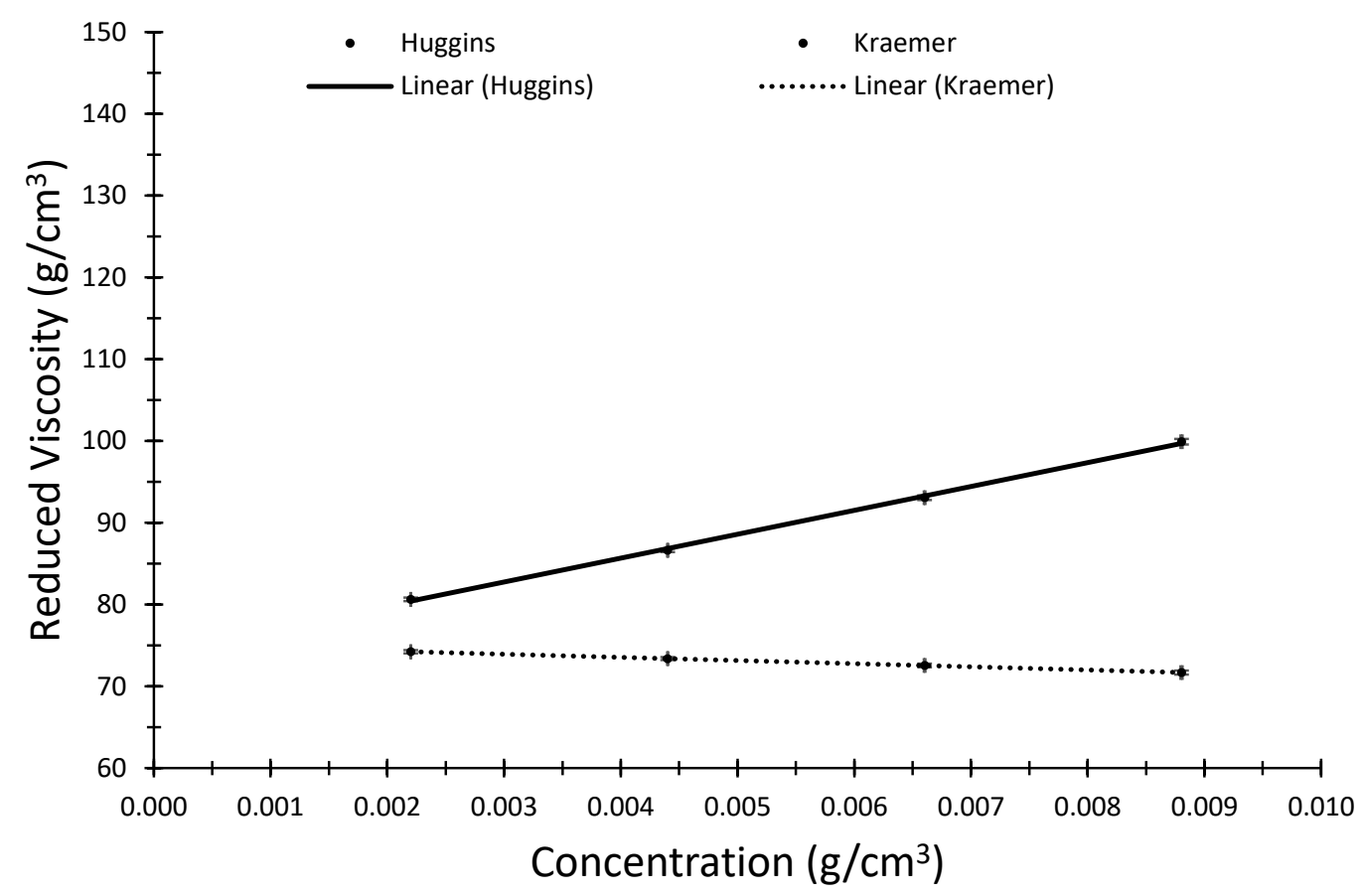

Figure A.6: EC45 Dilution at $100^{\circ} \mathrm{C}$

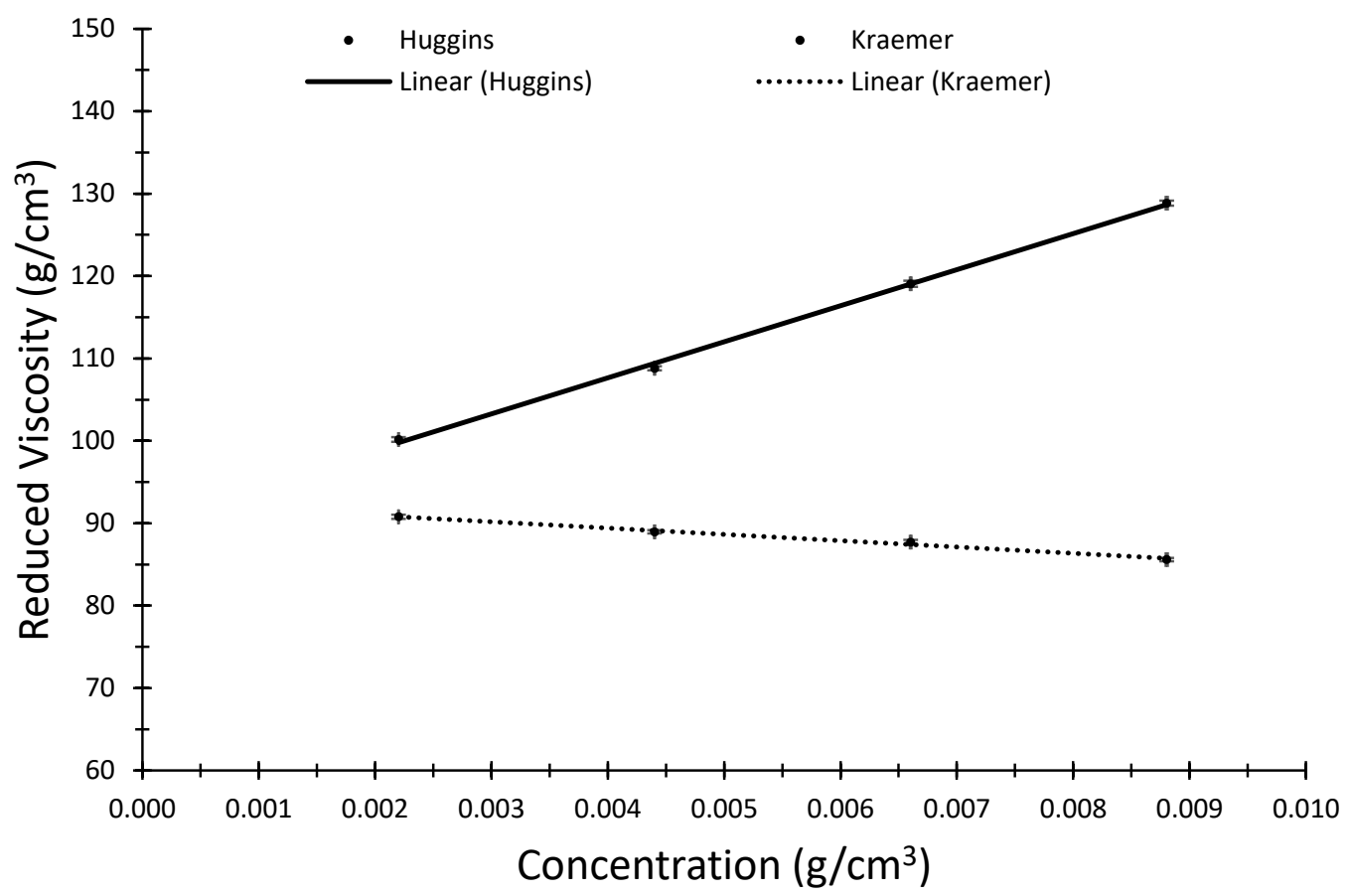

Figure A.7: EC45 30SC Dilution at $40^{\circ} \mathrm{C}$ 


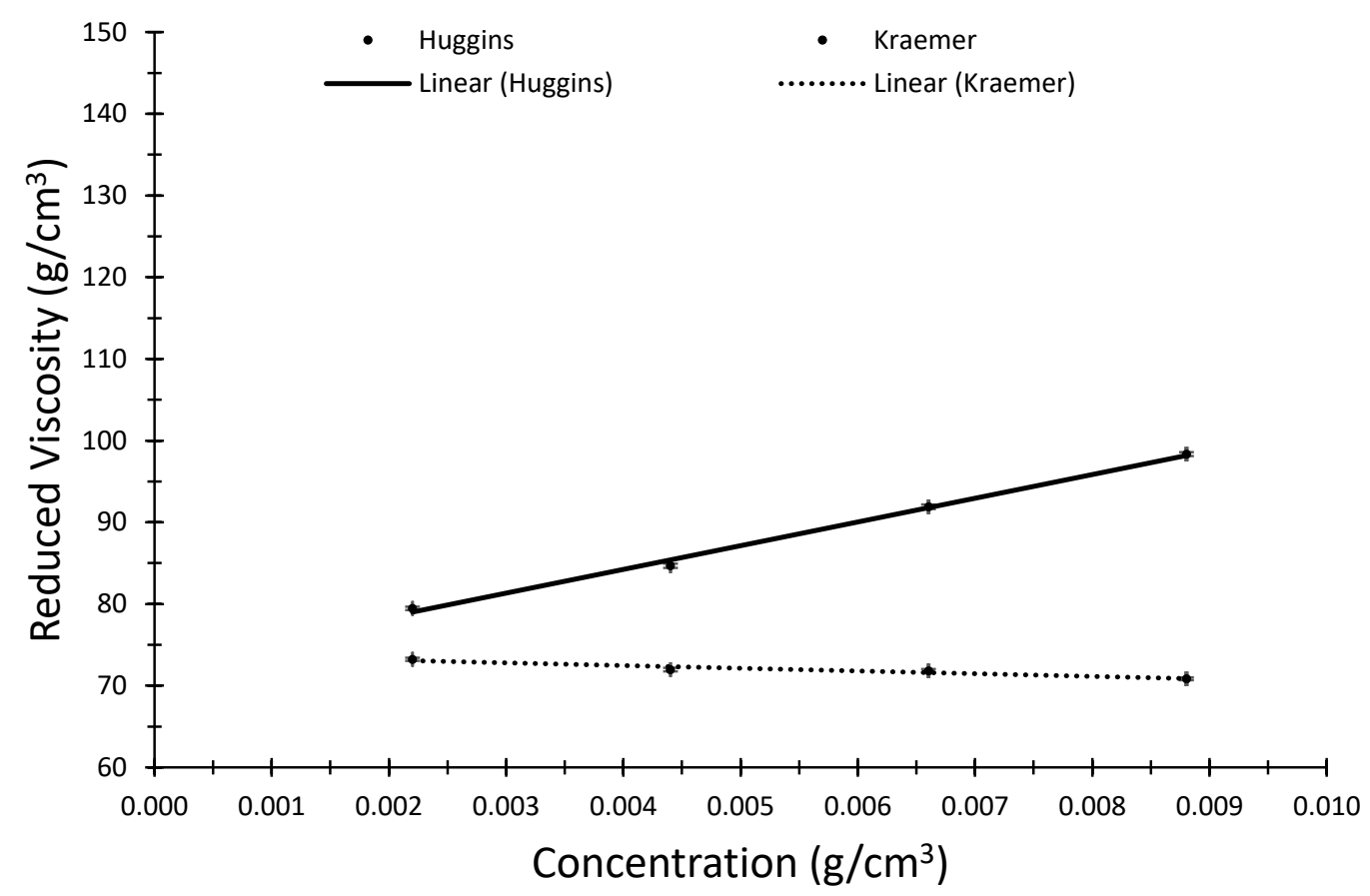

Figure A.8: EC45 30SC Dilution at $100^{\circ} \mathrm{C}$ 


\section{Appendix B}

\section{MATLAB Curve Fit}

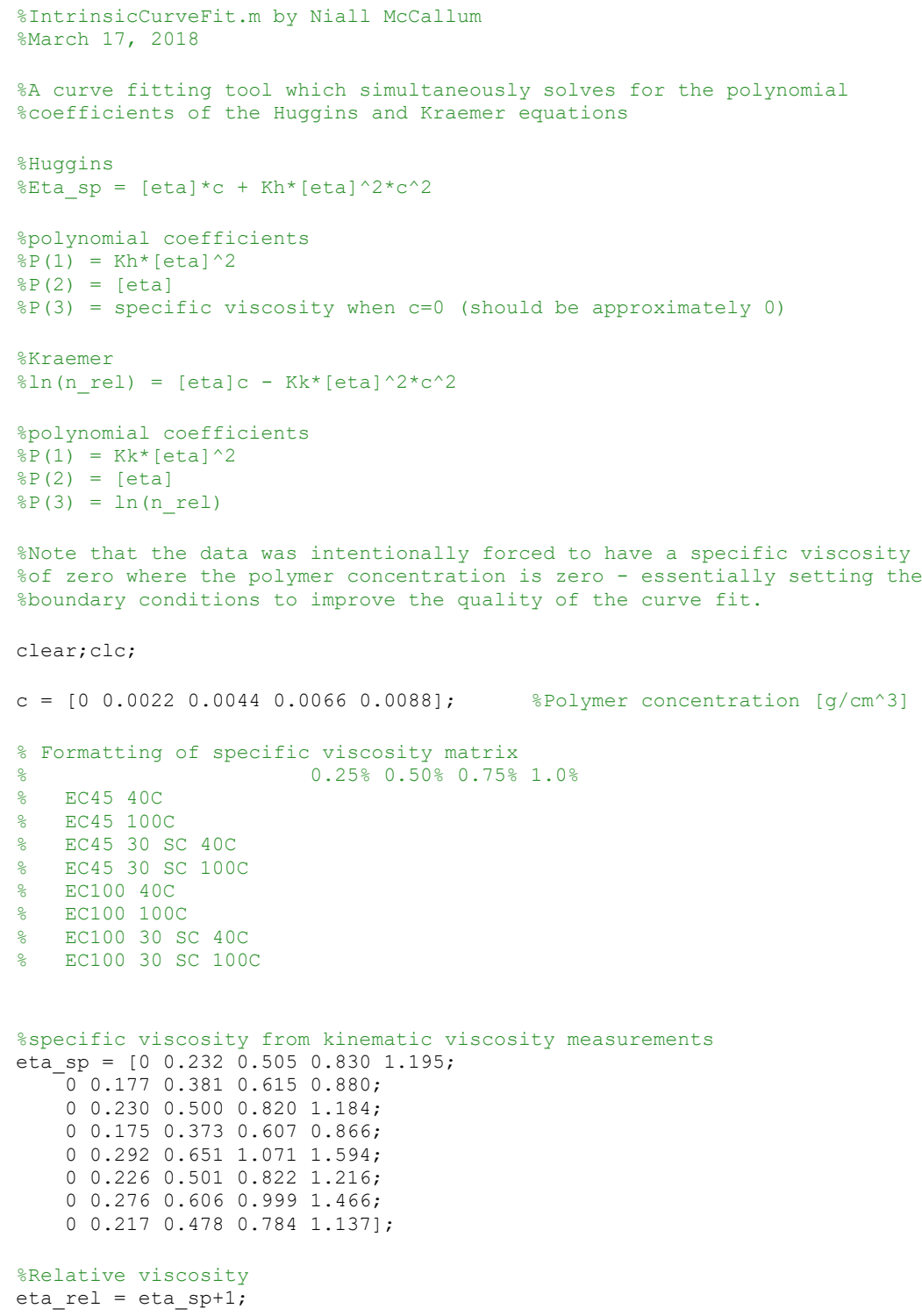




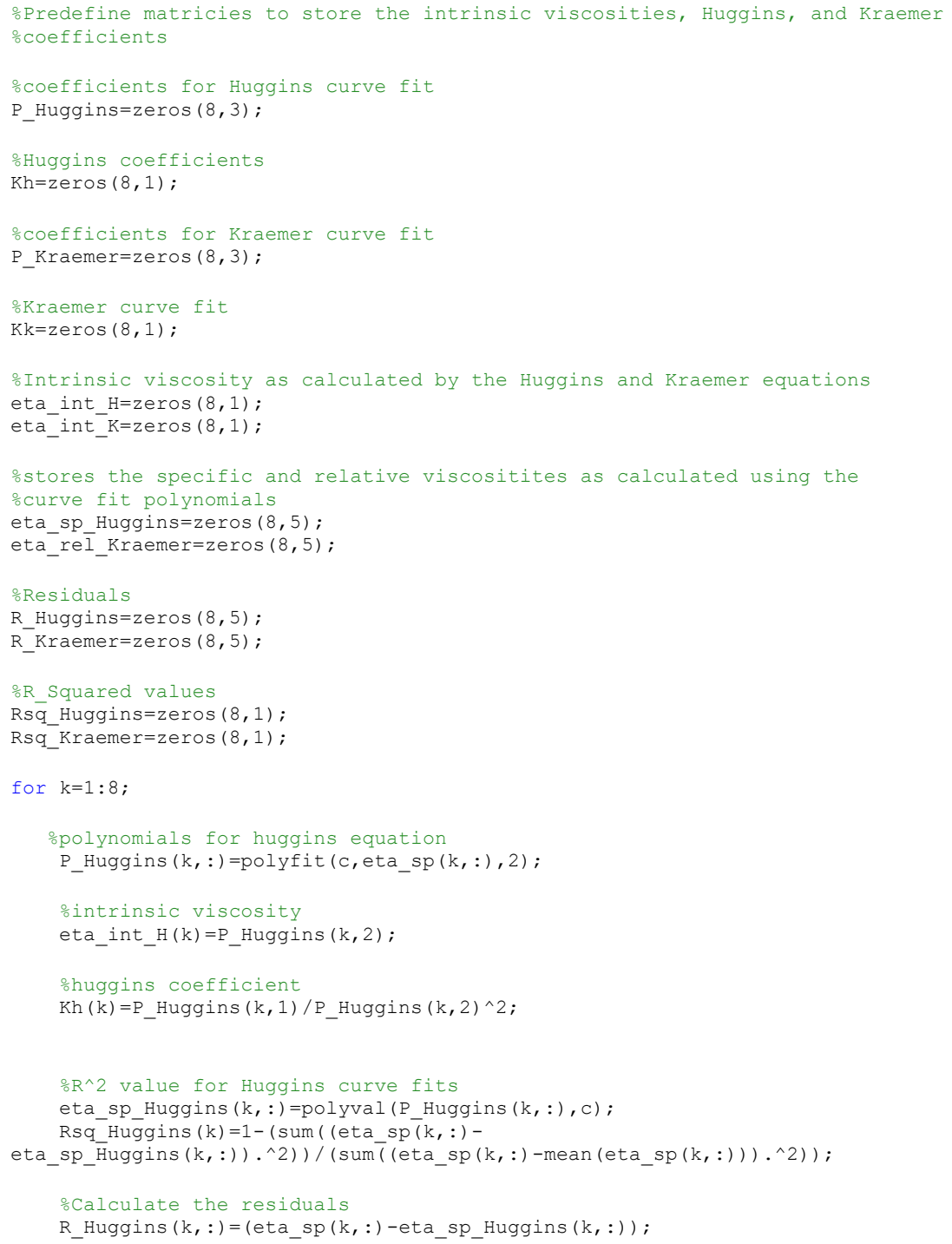


opolynomials for kraemer equation

P_Kraemer $(k,:)=\operatorname{polyfit}(c, \log ($ eta_rel $(k,:)), 2)$;

ointrinsic viscosity for kraemer equation

eta_int_K $(\mathrm{k})=$ P_Kraemer $(\mathrm{k}, 2)$;

okraemer coefficient

$\operatorname{Kk}(\mathrm{k})=\mathrm{P}_{-} \operatorname{Kraemer}(\mathrm{k}, 1) / \mathrm{P}_{-} \operatorname{Kraemer}(\mathrm{k}, 2)^{\wedge} 2$;

$\% \mathrm{R}^{\wedge} 2$ values for kraemer curve fits

eta rel $\operatorname{Kraemer}(\mathrm{k},:)=\exp (\operatorname{polyval}(\mathrm{P} \operatorname{Kraemer}(\mathrm{k},:), \mathrm{c}))$;

Rsq_Kraēerer $(k)=1-(\operatorname{sum}(($ eta_rel $(k,:) \overline{-}$

eta_rel_Kraemer $(k,:)) \cdot \wedge 2)) /\left(\operatorname{sum}\left(\left(\operatorname{eta} \_r e l(k,:)-\operatorname{mean}(\operatorname{eta} r r e l(k,:))\right) \cdot{ }^{\wedge}\right)\right)$;

oCalculate the residuals

R_Kraemer $(k,:)=($ eta_rel $(k,:)$-eta_rel_Kraemer $(k,:))$;

end 